\title{
Supersymmetry of Affine Toda Models as Fermionic Symmetry Flows of the Extended mKdV Hierarchy
}

\author{
David M. SCHMIDTT \\ Instituto de Física Teórica, UNESP-Universidade Estadual Paulista, \\ Caixa Postal 70532-2, 01156-970, São Paulo, SP, Brasil \\ E-mail: david@ift.unesp.br, david.schmidtt@gmail.com
}

Received December 10, 2009, in final form May 19, 2010; Published online May 27, 2010

doi:10.3842/SIGMA.2010.043

\begin{abstract}
We couple two copies of the supersymmetric mKdV hierarchy by means of the algebraic dressing technique. This allows to deduce the whole set of $(N, N)$ supersymmetry transformations of the relativistic sector of the extended mKdV hierarchy and to interpret them as fermionic symmetry flows. The construction is based on an extended RiemannHilbert problem for affine Kac-Moody superalgebras with a half-integer gradation. A generalized set of relativistic-like fermionic local current identities is introduced and it is shown that the simplest one, corresponding to the lowest isospectral times $t_{ \pm 1}$ provides the supercharges generating rigid supersymmetry transformations in $2 \mathrm{D}$ superspace. The number of supercharges is equal to the dimension of the fermionic kernel of a given semisimple element $E \in \widehat{\mathfrak{g}}$ which defines both, the physical degrees of freedom and the symmetries of the model. The general construction is applied to the $N=(1,1)$ and $N=(2,2)$ sinh-Gordon models which are worked out in detail.
\end{abstract}

Key words: algebraic dressing method; supersymmetry flows; supersymmetric affine Toda models

2010 Mathematics Subject Classification: 81T60; 37K20; 37K10

\section{Introduction}

It is well known that bosonic Toda models are underlined by Lie algebras and that they provide some sort of field theoretic realization to them. They are relevant to particle physics because they describe integrable perturbations of two-dimensional conformal field theories, allow soliton configurations in their spectrum and are useful laboratories to develop new methods relevant to the study of non-perturbative aspects of quantum field theory.

A natural step when having a bosonic field theory is to try to incorporate fermions and to construct its supersymmetry extension. In the case of bosonic Toda models this is a not an easy task because we want to preserve the integrability, which is one of the main properties of this kind of theories. Integrability is a consequence of the existence of an infinite number of bosonic Hamiltonians in involution which depend strongly on the Lie algebraic input data defining the Toda model itself. Each Hamiltonian generates a bosonic (even) symmetry flow and due to the fact that supersymmetry is just a symmetry, it is natural to expect the presence of conserved supercharges each one generating its own fermionic (odd) symmetry flow and also to expect that the supersymmetric extension is not related to a Lie algebra but to a Lie superalgebra, see [12] for an example of how bosonic symmetries are not preserved after supersymmetrization. By definition, a supersymmetry is a symmetry where the application of two successive odd transformations close into an even one. If there is an infinite number of even flows, then it is natural to incorporate the same number of odd flows in order to close the 'flow superalgebra'. Hence, the set of fields $\mathcal{F}$ will depend on an infinite number of even and odd variables $\mathcal{F}=$ $\mathcal{F}\left(t_{ \pm 1 / 2}, t_{ \pm 1}, t_{ \pm 3 / 2}, t_{ \pm 3}, \ldots\right)$, see [20] for a first example of this 'flow approach' applied to the KP 
hierarchy. Our main motivation to formulate supersymmetric affine Toda models within this setting relies on the possibility of using powerful techniques available in the theory of infinitedimensional Lie algebras and integrable systems, in particular, vertex operator representations and tau functions. The goal is to set the ground to study the quantization of the affine super Toda integrable models within this fashion.

Several authors have studied the problem of constructing supersymmetric extensions of integrable hierarchies. On one side, for the Toda lattice most of them use superfields as a natural way to supersymmetrize Lax operators while preserving integrability or to obtain a manifestly supersymmetric Hamiltonian reduction of super WZNW models, see for example [22, 11, 10, 5]. The common conclusion is that only Lie superalgebras (classical or affine) with a purely fermionic simple root system allow supersymmetric integrable extensions, otherwise supersymmetry is broken. On the other side, there are several supersymmetric formulations of the Drinfeld-Sokolov reduction method for constructing integrable hierarchies in which the algebraic Dressing method and the 'flow approach' were gradually developed and worked out in several examples, see for example $[18,9,19,4]$. The main goal of these works is the construction of an infinite set of fermionic non-local symmetry flows but a clear relation between the conserved supercharges and its corresponding field component transformations remains obscure. In [3], fermionic fields were coupled to the Toda fields in a supersymmetric way in the spirit of generalized Toda models coupled to matter fields introduced in [14] and further analyzed in [13]. This coupling was performed on-shell and only the first half of the supersymmetric sector was analyzed (corresponding to the positive part $\left.t_{+1 / 2}, t_{+1}\right)$. An important result of that paper was the introduction of a 'reductive' automorphism $\tau_{\text {red }}$ (constructed explicitly in the $s l(2,1)$ affine case) devised to remove the non-locality of the lowest supersymmetric flow $t_{+1 / 2}$, as a consequence, it was shown that it is not strictly necessary to start with an affine superalgebra with a purely fermionic simple root system in order to get an integrable supersymmetric extension of a bosonic model. See also [24] for another (based on $\operatorname{Osp}(1,4)$ having one bosonic and one fermionic simple roots) example of a Toda model with superconformal symmetry realized non-linearly. The complementary off-shell Hamiltonian reduction was developed in [15] by using a two-loop super-WZNW model where the (local) action functional leading to the supersymmetric Leznov-Saveliev equations of motion was constructed, in principle, for any superalgebra endowed with a half-integer gradation and invariant under $\tau_{\text {red }}$. It was also shown that several known purely fermionic integrable models belong to the family of perturbed WZNW on supercosets where the bosonic part is fully gauged away.

The purpose of this paper is to introduce the second half of the supersymmetric sector (corresponding to the negative part $t_{-1 / 2}, t_{-1}$ ) and to study the whole coupled system generated by the subset of symmetry flows $\left(t_{-1}, t_{-1 / 2}, t_{+1 / 2}, t_{+1}\right)$. This analysis was not performed neither in [3] nor [15] so this work complement their study. The outcome is that the supersymmetry flows described in terms of the algebraic dressing technique turn out to be equivalent to the usual notion of supersymmetry described in terms of superspace variables (this is shown by considering explicit examples). This allows to locate the supersymmetry of the models inside a formalism which is manifestly integrable by construction.

In Section 2.1 we review the algebraic dressing technique and use it to couple two identical copies of the same integrable hierarchy thus defining its extension. In Section 2.2 we introduce the relativistic/supersymmetric sector of the extended super-mKdV hierarchy by coupling two super-mKdV hierarchies in different gauges. This idea was first used in [21] in the bosonic case. In Section 2.3 we construct two odd Lax pairs associated to the chiral sectors of the hierarchy and in Section 2.4 the complete set of extended $(N, N)$ supersymmetry transformations is given. The recursion operators are given in Section 2.5 to show that all higher fermionic flows are non-local. In Section 2.6 we use the extended Riemann-Hilbert problem to construct a set of local fermionic current identities associated to the non-Abelian flows $t_{ \pm 1 / 2}$, where each pair of 
isospectral flows $t_{ \pm n}$ is coupled in a relativistic-like manner. It is also shown that the number of supersymmetries (i.e. supercharges) is equal to the dimension of the fermionic kernel of the operator $\operatorname{Ad} E$. This means that in the superalgebra decomposition $\widehat{\mathfrak{g}}=\operatorname{ker}(\operatorname{Ad} E) \oplus \operatorname{Im}(\operatorname{Ad} E)$ induced by the constant semisimple element $E$, any element of the affine superalgebra $\widehat{\mathfrak{g}}$ have a well defined role, i.e. it defines a symmetry flow or a physical degree of freedom. In Section 2.7 we use the two-Loop super WZNW action to construct the supercharges generating the supersymmetry transformations giving a direct relation between the $t_{ \pm 1 / 2}$ odd flows and the fields transformations. We also show that the Noether procedure reproduces the supercharges constructed in Section 2.6 by using the factorization problem thus confirming their equivalence. Finally, in the Sections 3.1 and 3.2 we study in detail the construction in order to have a better feeling of how the fermionic symmetry flows of the models are defined by the kernel part $(\operatorname{ker}(\operatorname{Ad} E))$ and to make contact with the usual notion of superspace. We also give an example of a solution to a relativistic-like equation expressed in terms of the higher graded $t_{ \pm 3}$ isospectral times only, thus generalizing the sine-Gordon equation. In the conclusion we pose the more important problems to be treated in the future which are the main motivations of the present work.

\section{General analysis}

Here we study the supersymmetric sector of the extended mKdV hierarchy and obtain the main results of the paper. The goal of this chapter is to put into one consistent body the new pieces with the known previous results. The core of the flow approach we will follow relies on the algebraic dressing technique used to unify symmetry flows (isospectral and non-Abelian) of integrable hierarchies related to affine Lie algebras. The Riemann-Hilbert factorization defines the integrable structure and a related hierarchy of non-linear partial differential equations.

\subsection{The algebraic dressing technique}

Consider an affine Lie superalgebra $\widehat{\mathfrak{g}}=\bigoplus_{i \in \mathbb{Z} / 2=-\infty}^{+\infty} \widehat{\mathfrak{g}}_{i}$ half-integer graded by an operator $Q$ $\left(\left[Q, \widehat{\mathfrak{g}}_{i}\right]=i \widehat{\mathfrak{g}}_{i}\right)$ and two supergroup elements (dressing matrices) $\Theta$ and $\Pi$ taken as the exponentials of the negative/positive subalgebras of $\widehat{\mathfrak{g}}$ respectively, i.e. $\widehat{\mathfrak{g}}_{-}$and $\widehat{\mathfrak{g}}_{+}$in the decomposition $\widehat{\mathfrak{g}}=\widehat{\mathfrak{g}}_{-}+\widehat{\mathfrak{g}}_{+}$induced by the projections $\mathcal{P}_{ \pm}(*)=(*)_{ \pm}$along positive and negative grades. They are taken to be formal expansions of the form

$$
\begin{aligned}
& \Theta=\exp \left(\psi^{(-1 / 2)}+\psi^{(-1)}+\psi^{(-3 / 2)}+\cdots\right), \\
& \Pi=B M, \quad M=\exp \left(-\psi^{(+1 / 2)}-\psi^{(+1)}-\psi^{(+3 / 2)}-\cdots\right),
\end{aligned}
$$

where $B=\exp \widehat{\mathfrak{g}}_{0} \in \widehat{G}_{0}$ and $\psi^{(i)} \in \widehat{\mathfrak{g}}_{i}$. The constant semisimple elements $E^{( \pm 1)}$ of grade \pm 1 $\left(\left[Q, E^{(+1)}\right]= \pm E^{(+1)}\right)$ define operators $\operatorname{Ad} E^{( \pm 1)}$ each one splitting the superalgebra $\widehat{\mathfrak{g}}=\mathcal{K}^{ \pm}+$ $\mathcal{M}^{ \pm}$into kernel and image subspaces obeying $\left[\mathcal{K}^{ \pm}, \mathcal{K}^{ \pm}\right] \subset \mathcal{K}^{ \pm},\left[\mathcal{K}^{ \pm}, \mathcal{M}^{ \pm}\right] \subset \mathcal{M}^{ \pm}$, where $\mathcal{K}^{ \pm} \equiv$ $\operatorname{ker}\left(\operatorname{Ad} E^{( \pm 1)}\right)$ and $\mathcal{M}^{ \pm} \equiv \operatorname{Im}\left(\operatorname{Ad} E^{( \pm 1)}\right)$. The kernel and image subspaces have bosonic and fermionic components $\mathcal{K}^{ \pm}=\mathcal{K}_{B}^{ \pm} \oplus \mathcal{K}_{F}^{ \pm}$and $\mathcal{M}^{ \pm}=\mathcal{M}_{B}^{ \pm} \oplus \mathcal{M}_{F}^{ \pm}$each one having a well defined (half-integer) grade respect the operator $Q$.

Recall [6] that the dressing transformation of $x \in \widehat{G}$ by $g \in \widehat{G}$ is defined by

$$
{ }^{g} x=\left(x g x^{-1}\right)_{ \pm} x g_{ \pm}^{-1} .
$$

The infinitesimal transformation for $g=\exp A$ with $A=A_{+}+A_{-}$and $A_{ \pm} \in \widehat{\mathfrak{g}}_{ \pm}$is

$$
\delta_{A} x={ }^{g} x-x= \pm\left(x A x^{-1}\right)_{ \pm} x \mp x A_{ \pm} .
$$


From this we find the pure actions of $A=A_{+} \in \mathcal{K}_{+}$and $A=A_{-} \in \mathcal{K}_{-}$on $x=\Theta$ and $x=\Pi$, respectively

$$
\delta_{A_{+}} \Theta=-\left(\Theta A_{+} \Theta^{-1}\right)_{-} \Theta, \quad \delta_{A_{-}} \Pi=+\left(\Pi A_{-} \Pi^{-1}\right)_{+} \Pi .
$$

To see this, consider $A=A_{+}$and $x=\Theta$ and the upper sign in (2). We get

$$
\delta_{A_{+}} \Theta=\left(\Theta A_{+} \Theta^{-1}\right)_{+} \Theta-\Theta A_{+}=-\left(\Theta A_{+} \Theta^{-1}\right)_{-} \Theta,
$$

where we have used the decomposition $\Theta A_{+} \Theta^{-1}=\left(\Theta A_{+} \Theta^{-1}\right)_{+}+\left(\Theta A_{+} \Theta^{-1}\right)_{-}$. For $A=A_{-}$ and $x=\Pi$ the proof is similar. We also have that for $A=A_{-}$and $x=\Theta$ and for $A=A_{+}$and $x=\Pi$ the variations vanish, $\delta_{A_{-}} \Theta=0$ and $\delta_{A_{+}} \Pi=0$ respectively. Hence, in the present form, the dressing matrices (1) only evolve under half of the flows.

Setting $A_{ \pm}=t_{ \pm n} E^{( \pm n)}$ (with $\left[Q, E^{( \pm n)}\right]= \pm n E^{( \pm n)}$ ) and taking the limit $t_{ \pm n} \rightarrow 0$, we have the isospectral evolutions for $\Theta$ and $\Pi$

$$
\partial_{+n} \Theta=-\left(\Theta E^{(+n)} \Theta^{-1}\right)_{-} \Theta, \quad \partial_{-n} \Pi=+\left(\Pi E^{(-n)} \Pi^{-1}\right)_{+} \Pi,
$$

where $\delta_{A_{+}} \Theta / t_{+}=\left(A_{+} \Theta-\Theta\right) / t_{+} \rightarrow \partial_{+n} \Theta$ and similar for $\delta_{A_{-}} \Pi$. From equations (4) we obtain the dressing relations

$$
\begin{aligned}
& E_{\Theta}^{(+n)}=\left(\Theta E^{(+n)} \Theta^{-1}\right)_{+}=\Theta E^{(+n)} \Theta^{-1}+\partial_{+n} \Theta \Theta^{-1}, \\
& E_{\Pi}^{(-n)}=\left(\Pi E^{(-n)} \Pi^{-1}\right)_{-}=\Pi E^{(-n)} \Pi^{-1}-\partial_{-n} \Pi \Pi^{-1}
\end{aligned}
$$

and the Lax operators

$$
L_{+n}=\partial_{+n}-E_{\Theta}^{(+n)}, \quad L_{-n}=\partial_{-n}+E_{\Pi}^{(-n)} .
$$

The Baker-Akhiezer wave functions $\Psi_{ \pm}$are defined by $L_{ \pm n} \Psi_{\mp}=0$ and are given by

$$
\Psi_{-}=\Theta \exp \left(+\sum_{n \in \mathbb{Z}^{+}} t_{+n} E^{(+n)}\right), \quad \Psi_{+}=\Pi \exp \left(-\sum_{n \in \mathbb{Z}^{+}} t_{-n} E^{(-n)}\right) .
$$

Equations (4) describe two identical but decoupled systems of evolution equations as shown above, the coupling of the two sectors is achieved by imposing the relation $g=\Psi_{-}^{-1} \Psi_{+}$with $g$ a constant group element. Alternatively, we have

$$
\exp \left(+\sum_{n \in \mathbb{Z}^{+}} t_{+n} E^{(+n)}\right) g \exp \left(+\sum_{n \in \mathbb{Z}^{+}} t_{-n} E^{(-n)}\right)=\Theta^{-1}(t) \Pi(t) .
$$

This is the extended Riemann-Hilbert factorization problem originally used in [2] to extend the $\mathrm{mKdV}$ hierarchy to the negative flows. From (5) we recover (4) and two important extra equations describing the isospectral evolution of $\Theta$ and $\Pi$ with respect opposite flow parameters

$$
\partial_{+n} \Pi=+\left(\Theta E^{(+n)} \Theta^{-1}\right)_{+} \Pi, \quad \partial_{-n} \Theta=-\left(\Pi E^{(-n)} \Pi^{-1}\right)_{-} \Theta .
$$

These equations are extended to actions of $A_{+} \in \mathcal{K}_{+}$and $A_{-} \in \mathcal{K}_{-}$on $\Pi$ and $\Theta$, similar to (3) we have

$$
\delta_{A_{+}} \Pi=+\left(\Theta A_{+} \Theta^{-1}\right)_{+} \Pi, \quad \delta_{A_{-}} \Theta=-\left(\Pi A_{-} \Pi^{-1}\right)_{-} \Theta .
$$

The equations (3), (4) and (6), (7) describe the isospectral evolution and non-Abelian variations of the dressing matrices $\Theta$ and $\Pi$ and their consistency, as an algebra of flows, is encoded in Proposition 1 below. Note that the flows associated to the positive times are dual to the ones associated to the negative times, in the sense that $\mathcal{K}_{+}^{*} \simeq \mathcal{K}_{-}$under the (assumed to exists) nondegenerate inner product $\langle *\rangle$ which provide the orthogonality condition $\left\langle\widehat{\mathfrak{g}}_{i} \widehat{\mathfrak{g}}_{j}\right\rangle=\delta_{i+j, 0}$ of graded spaces. This also show how the degrees of freedom are naturally doubled by the extension. 
Remark 1. If we consider pseudo-differential operators, the equations (4), (6) are good starting points to extend the KP hierarchy with the negative flows and the expectation value of (5) to extend its corresponding $\tau$-function.

\subsection{Relativistic sector of the extended $\mathrm{mKdV}$ hierarchy}

From (4) and (6) we have the following

Definition 1. The relativistic sector of the extended $m K d V$ hierarchy is defined by the following set of evolution equations

$$
\begin{array}{ll}
\partial_{+} \Theta=+\left(\Theta E_{+}^{(+1)} \Theta^{-1}\right)_{<0} \Theta, & \partial_{+} \Pi=-\left(\Theta E_{+}^{(+1)} \Theta^{-1}\right)_{\geq 0} \Pi, \\
\partial_{-} \Theta=+\left(\Pi E_{-}^{(-1)} \Pi^{-1}\right)_{<0} \Theta, & \partial_{-} \Pi=-\left(\Pi E_{-}^{(-1)} \Pi^{-1}\right)_{\geq 0} \Pi,
\end{array}
$$

for the two isospectral times $t_{ \pm 1}=-x^{ \pm}$associated to the grade \pm 1 constant elements $E_{ \pm}^{( \pm 1)} \in \widehat{\mathfrak{g}}$ The $(*)_{\geq 0}$ denote projection onto grades $\geq 0$ and the $(*)_{<0}$ onto grades $\leq-1 / 2$.

In the definition above we write explicitly the projections $(*)_{ \pm}$in terms of grades in order to avoid confusion with the different projections used below in (10). The Lax covariant derivative $\left(L=d+A^{L}\right)$ extracted from (8) has a Lax connection $A^{L}$ given by

$$
\begin{array}{ll}
L_{-}=\partial_{-}+A_{-}^{L}, & A_{-}^{L}=-B\left(E_{-}^{(-1)}+\psi_{-}^{(-1 / 2)}\right) B^{-1}, \\
L_{+}=\partial_{+}+A_{+}^{L}, & A_{+}^{L}=-\partial_{+} B B^{-1}+\psi_{+}^{(+1 / 2)}+E_{+}^{(+1)},
\end{array}
$$

where

$$
\psi_{ \pm}^{( \pm 1 / 2)}= \pm\left[\psi^{(\mp 1 / 2)}, E_{ \pm}^{( \pm 1)}\right] \in \mathcal{M}_{F}^{( \pm 1 / 2)} .
$$

The RHS of (5) can be written in an equivalent way because we have

$$
\Theta^{-1}(t) \Pi(t)=\Theta^{-1}(t) B M=\left(B^{-1} \Theta\right)^{-1} M
$$

and this motivates the following

Definition 2. The gauge-equivalent relativistic sector is defined by the following set of evolution equations

$$
\begin{array}{ll}
\partial_{+} \Theta^{\prime}=+\left(\Theta^{\prime} E_{+}^{(+1)} \Theta^{\prime-1}\right)_{\leq 0} \Theta^{\prime}, & \partial_{+} \Pi^{\prime}=-\left(\Theta^{\prime} E_{+}^{(+1)} \Theta^{\prime-1}\right)_{>0} \Pi^{\prime}, \\
\partial_{-} \Theta^{\prime}=+\left(\Pi^{\prime} E_{-}^{(-1)} \Pi^{\prime-1}\right)_{\leq 0} \Theta^{\prime}, & \partial_{-} \Pi^{\prime}=-\left(\Pi^{\prime} E_{-}^{(-1)} \Pi^{\prime-1}\right)_{>0} \Pi^{\prime},
\end{array}
$$

where $\Theta^{\prime}=B^{-1} \Theta$ and $\Pi^{\prime}=M$. The $(*)_{>0}$ denote projection onto grades $\geq+1 / 2$ and $(*)_{\leq 0}$ onto grades $\leq 0$.

The Lax covariant derivative extracted from (10) has a Lax connection

$$
\begin{array}{ll}
L_{-}^{\prime}=\partial_{-}+A_{-}^{L L}, & A_{-}^{\prime L}=B^{-1} \partial_{-} B-\psi_{-}^{(-1 / 2)}-E_{-}^{(-1)}, \\
L_{+}^{\prime}=\partial_{+}+A_{+}^{L L}, & A_{+}^{\prime L}=B^{-1}\left(E_{+}^{(+1)}+\psi_{+}^{(+1 / 2)}\right) B
\end{array}
$$

and it is related to (9) by a gauge transformation $L \rightarrow L^{\prime}$, where $A^{L}=B^{-1} A^{L} B-d B^{-1} B$. Clearly, the two definitions are equivalent.

The constant part of the Lax connection is given by ( $\Sigma$ is parametrized by $x^{ \pm}$)

$$
E^{( \pm 1)}=E_{ \pm}^{( \pm 1)} d x^{ \pm} \in \Omega_{B}(\Sigma) \otimes \widehat{\mathfrak{g}}_{( \pm 1)}
$$


and change under coordinate transformations because of their $d x^{ \pm}$basis. We also have that

$$
\psi_{ \pm}^{( \pm 1 / 2)} d x^{ \pm}=\Omega_{F}(\Sigma) \otimes \widehat{\mathfrak{g}}_{( \pm 1 / 2)}
$$

are fermionic 1-forms. Thus, $A^{L}$ is a superalgebra-valued 1 -form. This is to recall that no superspace formulation is involved in the construction of our super-Lax operators and that the approach relies entirely on pure Lie algebraic properties.

The equations of motion are defined by the zero curvature of $A^{L}$, namely $\left[L_{+}, L_{-}\right]=0$ and leads to a system of non-linear differential equations in which the derivatives $\partial_{ \pm}$appear mixed with the same order, hence the name relativistic. The coupling of one positive and one negative higher graded isospectral flow of opposite sign is direct from the construction. This allows the construction of relativistic-like integrable equations, see equation (37) below for an example.

In the definitions of the Lax operators above we actually have

$$
-\partial_{+} B B^{-1}=A_{+}^{(0)}+Q_{+}^{(0)}, \quad-B^{-1} \partial_{-} B=A_{-}^{(0)}+Q_{-}^{(0)},
$$

where (the upper label denoting the $Q$ grading)

$$
A_{ \pm}^{(0)}= \pm\left[\psi^{(\mp 1)}, E_{ \pm}^{( \pm 1)}\right] \in \mathcal{M}_{B}^{(0)}, \quad Q_{ \pm}^{(0)}=\frac{1}{2}\left[\psi^{(\mp 1 / 2)},\left[\psi^{(\mp 1 / 2)}, E_{ \pm}^{( \pm 1)}\right]\right] \in \mathcal{K}_{B}^{(0)}
$$

These relations are the solutions to the grade -1 and +1 components of the zero curvature relations $\left[L_{+}, L_{-}\right]_{-1}=\left[L_{+}^{\prime}, L_{-}^{\prime}\right]_{+1}=0$ for the operators $L_{ \pm}$and $L_{ \pm}^{\prime}$ obtained from (8) and (10).

The presence of the fermion bilinear $Q_{ \pm}^{(0)}$ results in the non-locality of the odd $t_{ \pm 1 / 2}$ symmetry flows [3] and also in the existence of gauge symmetries of the models as can be deduced from the off-shell formulation of the system (9) done in [15]. Having $\mathcal{K}_{B}^{(0)} \neq \varnothing$ translates into the existence of flat directions of the Toda potential which takes the models out of the $\mathrm{mKdV}$ hierarchy. Thus, we impose the vanishing of $Q_{ \pm}^{(0)}$. Another reason why we impose $Q_{ \pm}^{(0)}=0$, is to get a well defined relation between the dressing matrix $\Theta$ and the term $A_{ \pm}^{(0)}$ in the spirit of [4], which means that the dynamical fields are described entirely in terms of the image part of the algebra $\mathcal{M}$. The kernel part $\mathcal{K}$ is responsible only for the symmetries of the model and all this together clarifies the role played by the term $Q_{ \pm}^{(0)}$. Then, by restricting to superalgebras in which $Q_{ \pm}^{(0)}=0$ we have local $t_{ \pm 1 / 2}$ flows and models inside the $\mathrm{mKdV}$ hierarchy.

Remark 2. Flat directions in the Toda potential $V_{B}=\left\langle E_{+}^{(+1)} B E_{-}^{(-1)} B^{-1}\right\rangle$ allows the existence of soliton solutions with Noether charges, e.g. the electrically charged solitons of the complex sine-Gordon model which is known to belong to the relativistic sector of the AKNS hierarchy [1] instead of the $\mathrm{mKdV}$.

We parametrize the Toda field as $B=g \exp [\eta Q] \exp [\nu C]$, provided we have a subalgebra solution to the algebraic conditions $Q_{ \pm}^{(0)}=0$. The model is then defined on a reduced group manifold and (11) is conveniently parametrized in the image part $\mathcal{M}_{B}^{(0)}$ of the algebra, i.e. $-\partial_{+} B B^{-1}=A_{+}^{(0)}$ and $-B^{-1} \partial_{-} B=A_{-}^{(0)}$.

The zero curvature $\left(F_{L}=0\right)$ of (9) gives the supersymmetric version of the Leznov-Saveliev equations [3]

$$
\begin{aligned}
& 0=F_{+-}^{(+1 / 2)}=-\partial_{-} \psi_{+}^{(+1 / 2)}+\left[B \psi_{-}^{(-1 / 2)} B^{-1}, E_{+}^{(+1)}\right], \\
& 0=F_{+-}^{(0)}=\partial_{-}\left(\partial_{+} B B^{-1}\right)-\left[E_{+}^{(+1)}, B E_{-}^{(-1)} B^{-1}\right]-\left[\psi_{+}^{(+1 / 2)}, B \psi_{-}^{(-1 / 2)} B^{-1}\right], \\
& 0=F_{+-}^{(-1 / 2)}=B\left(-\partial_{+} \psi_{-}^{(-1 / 2)}+\left[E_{-}^{(-1)}, B^{-1} \psi_{+}^{(+1 / 2)} B\right]\right) B^{-1} .
\end{aligned}
$$


Written more explicitly in the form

$$
\begin{aligned}
& \partial_{-} \psi_{+}^{(+1 / 2)}=e^{-\eta / 2}\left[g \psi_{-}^{(-1 / 2)} g^{-1}, E_{+}^{(+1)}\right], \\
& \partial_{-}\left(\partial_{+} g g^{-1}\right)+\partial_{-} \partial_{+} \nu=e^{-\eta}\left[E_{+}^{(+1)}, g E_{-}^{(-1)} g^{-1}\right]+e^{-\eta / 2}\left[\psi_{+}^{(+1 / 2)}, g \psi_{-}^{(-1 / 2)} g^{-1}\right], \\
& \partial_{+} \psi_{-}^{(-1 / 2)}=e^{-\eta / 2}\left[E_{-}^{(-1)}, g^{-1} \psi_{+}^{(+1 / 2)} g\right], \quad \partial_{-} \partial_{+} \eta Q=0,
\end{aligned}
$$

the linearized equations of motion with $\eta=\eta_{0}, \eta_{0} \in \mathbb{R}$ may be written in the Klein-Gordon form

$$
\left(\partial_{+} \partial_{-}+\widehat{m}^{2}\right) \circ(\Xi)=0, \quad \partial_{+} \partial_{-} \nu-\Lambda=0
$$

for $\Xi=\psi^{( \pm 1 / 2)}$ and $\log g$, where $\widehat{m}^{2}$ is the mass operator

$$
\widehat{m}^{2}(\Xi)=e^{-\eta_{0}}\left(\operatorname{ad} E_{-}^{(-1)} \circ \operatorname{ad} E_{+}^{(+1)}\right) \circ(\Xi)=m^{2} I \Xi .
$$

We have used $e^{-\eta_{0}}\left[E_{+}^{(+1)}, E_{-}^{(-1)}\right]=\Lambda C$. Then, the Higgs-like field $\eta_{0}$ sets the mass scale of the theory. The massless limit corresponds to $\eta_{0} \rightarrow \infty$. Note that all fields have the same mass which is what we would expect in a supersymmetric theory. Taking $\eta=\eta_{0}$, the free fermion equations of motion reads

$$
\partial_{ \pm} \psi^{( \pm 1 / 2)}=\mp \widehat{m}^{ \pm}\left(\psi^{(\mp 1 / 2)}\right)
$$

where $\widehat{m}^{ \pm}(*)=e^{-\eta_{0} / 2} \operatorname{ad} E_{ \pm}^{( \pm 1)} \circ(*)$. These equations show that fermions of opposite 'chirality' are mixed by the mass term and that in the massless limit they decouple. This means that positive/negative flows are naturally related to the two chiralities in the field theory. In most of the literature, only the positive set of times is usually considered.

The role of the fields $\nu$ and $\eta$ associated to the central term $C$ (of the Kac-Moody algebra $\widehat{\mathfrak{g}}$ ) and grading operator $Q$ is to restore the conformal symmetry of the models associated to the Loop algebra $\widetilde{\mathfrak{g}}$ (which are non-conformal) so we are actually dealing with conformal affine Toda models.

\subsection{Non-Abelian flows: the odd Lax pairs $L_{ \pm 1 / 2}$}

Here we deduce the two lowest odd degree fermionic Lax operators giving rise to the $\pm 1 / 2$ supersymmetry flows, which are the ones we are mainly concerned in the body of the paper. The negative part is the novelty here. From (3) and (7) we have

Definition 3. The non-Abelian evolution equations of the Dressing matrices are defined by

$$
\begin{array}{ll}
\delta_{K^{(+)}} \Theta=-\left(\Theta K^{(+)} \Theta^{-1}\right)_{<0} \Theta, & \delta_{K^{(+)}} \Pi=+\left(\Theta K^{(+)} \Theta^{-1}\right)_{\geq 0} \Pi, \\
\delta_{K^{(-)}} \Theta=-\left(\Pi K^{(-)} \Pi^{-1}\right)_{<0} \Theta, & \delta_{K^{(-)}} \Pi=+\left(\Pi K^{(-)} \Pi^{-1}\right)_{\geq 0} \Pi,
\end{array}
$$

for some positive/negative degree generators $K^{(+)}$and $K^{(-)}$in the kernel of the operators $\operatorname{Ad} E^{( \pm 1)}$. Equivalently, we have

$$
\begin{array}{ll}
\delta_{K^{(+)}} \Theta^{\prime}=-\left(\Theta^{\prime} K^{(+)} \Theta^{\prime-1}\right)_{\leq 0} \Theta^{\prime}, & \delta_{K^{(+)}} \Pi^{\prime}=+\left(\Theta^{\prime} K^{(+)} \Theta^{\prime-1}\right)_{>0} \Pi^{\prime}, \\
\delta_{K^{(-)}} \Theta^{\prime}=-\left(\Pi^{\prime} K^{(-)} \Pi^{\prime-1}\right)_{\leq 0} \Theta^{\prime}, & \delta_{K^{(-)}} \Pi^{\prime}=+\left(\Pi^{\prime} K^{(-)} \Pi^{\prime-1}\right)_{>0} \Pi^{\prime} .
\end{array}
$$

The consistency of all flows, as an algebra, is encoded in the following 
Proposition 1. The flows (13) and (14) satisfy

$$
\left[\delta_{K_{i}^{( \pm)}}, \delta_{K_{j}^{( \pm)}}\right](*)=\delta_{\mp\left[K_{i}^{( \pm)}, K_{j}^{( \pm)}\right]}(*), \quad\left[\delta_{K_{i}^{(+)}}, \delta_{K_{j}^{(-)}}\right](*)=0,
$$

where $(*)=\Theta, \Pi, \Theta^{\prime}, \Pi^{\prime}$. The map $\delta: \mathcal{K} \rightarrow \delta_{\mathcal{K}}$ is a homomorphism.

Proof. The proof is straightforward after noting that $[X, Y]_{ \pm}=\left[X_{ \pm}, Y_{ \pm}\right]+\left[X_{ \pm}, Y_{\mp}\right]_{ \pm}+$ $\left[X_{\mp}, Y_{ \pm}\right]_{ \pm}$

The last relation above means that the symmetries generated by elements in $\mathcal{K}_{ \pm}$commute themselves. This can be traced back to be a consequence of the second Lie structure induced on $\widehat{\mathfrak{g}}$ by the action of the dressing group which introduces a classical super $r$-matrix $R=\frac{1}{2}\left(\mathcal{P}_{+}-\mathcal{P}_{-}\right)$ defined in terms of the projections $\mathcal{P}_{+}$and $\mathcal{P}_{-}$of $\widehat{\mathfrak{g}}=\widehat{\mathfrak{g}}_{+}+\widehat{\mathfrak{g}}_{-}$along the positive/negative subalgebras $\widehat{\mathfrak{g}}_{ \pm}$, see also [9]. The map $\delta: \mathcal{K} \rightarrow \delta_{\mathcal{K}}$ is actually a map (up to a global irrelevant sign) to the $R$-bracket (see [7]) $\left[\delta_{K}, \delta_{K^{\prime}}\right]=\delta_{\left[K, K^{\prime}\right]_{R}}$, where $\left[K, K^{\prime}\right]_{R}=\left[K, R\left(K^{\prime}\right)\right]+\left[R(K), K^{\prime}\right]$. Hence, all the symmetries generated by $\mathcal{K}$ are chiral as a consequence of the second Lie structure. In particular, this imply the commutativity of the $2 \mathrm{D}$ rigid supersymmetry transformations cf. (20) below, as expected.

The $\pm 1 / 2$ flows are generated by the elements $\mp D^{( \pm 1 / 2)} \in \mathcal{K}_{F}^{( \pm 1 / 2)}$ of grades $\pm 1 / 2$ in the fermionic part of the kernel, where $D^{( \pm 1 / 2)}$ depend on the infinitesimal constant grassmannian parameters. They define the evolution equations (actually variations cf. (3), (7))

$$
\begin{array}{ll}
\delta_{+1 / 2} \Theta=+\left(\Theta D^{(+1 / 2)} \Theta^{-1}\right)_{<0} \Theta, & \delta_{+1 / 2} \Pi=-\left(\Theta D^{(+1 / 2)} \Theta^{-1}\right)_{\geq 0} \Pi, \\
\delta_{-1 / 2} \Theta=-\left(\Pi D^{(-1 / 2)} \Pi^{-1}\right)_{<0} \Theta, & \delta_{-1 / 2} \Pi=+\left(\Pi D^{(-1 / 2)} \Pi^{-1}\right)_{\geq 0} \Pi,
\end{array}
$$

giving rise to the dressing expressions

$$
\begin{aligned}
& \Theta\left(\delta_{+1 / 2}+D^{(+1 / 2)}\right) \Theta^{-1}=\delta_{+1 / 2}+D^{(0)}+D^{(+1 / 2)}=L_{+1 / 2}, \\
& \Pi\left(\delta_{-1 / 2}+D^{(-1 / 2)}\right) \Pi^{-1}=\delta_{-1 / 2}+B D^{(-1 / 2)} B^{-1}=L_{-1 / 2},
\end{aligned}
$$

where $D^{(0)}=\left[\psi^{(-1 / 2)}, D^{(+1 / 2)}\right] \in \mathcal{M}_{B}^{(0)}$. The derivation of $L_{-1 / 2}$ follows exactly the same lines for the derivation of $L_{+1 / 2}$ done in [3]. At this point we have four Lax operators $L_{ \pm 1 / 2}$ and $L_{ \pm 1}$. The grade subspace decomposition of the relations $\left[L_{ \pm 1 / 2}, L_{+1}\right]=\left[L_{ \pm 1 / 2}, L_{-1}\right]=0$ allows to take the solution $D^{(0)}=-\delta_{+1 / 2} B B^{-1}$. The compatibility of this system of four Lax operators provides the $2 \mathrm{D}$ supersymmetry transformations among the field components. Indeed, using the equations of motion we get their explicit form, see equation (17) and (18) below.

Finally, the odd Lax operators reads

$$
\begin{aligned}
& L_{+1 / 2}=\delta_{+1 / 2}-\delta_{+1 / 2} B B^{-1}+D^{(+1 / 2)}, \\
& L_{-1 / 2}=\delta_{-1 / 2}+B D^{(-1 / 2)} B^{-1} .
\end{aligned}
$$

The operator $L_{+1 / 2}$ was already constructed in [3] and the $L_{-1 / 2}$ is the novelty here.

Note that in (15) and (16) are in different gauges. This is the key idea for introducing the Toda potential (superpotential) in the supersymmetry transformations which is also responsible for coupling the two sectors.

\subsection{Local supersymmetry flows $\delta_{ \pm 1 / 2}$}

The equations (12) are invariant under a pair of non-Abelian fermionic flows $\left(\delta_{\mathrm{SUSY}}=\delta_{-1 / 2}+\right.$ $\left.\delta_{+1 / 2}\right)$ as a consequence of the compatibility relations $\left[L_{ \pm 1 / 2}, L_{+}\right]=\left[L_{ \pm 1 / 2}, L_{-}\right]=0$ supplemented by the equations of motion $\left[L_{+}, L_{-}\right]=0$ and the Jacobi identity. They are generated by the elements in the fermionic kernel $\mathcal{K}_{F}^{( \pm 1 / 2)}$ and are explicitly given by

$$
\delta_{+1 / 2} \psi_{-}^{(-1 / 2)}=\left[E_{-}^{(-1)}, B^{-1} D^{(+1 / 2)} B\right], \quad \delta_{+1 / 2} B B^{-1}=\left[D^{(+1 / 2)}, \psi^{(-1 / 2)}\right],
$$




$$
\delta_{+1 / 2} \psi_{+}^{(+1 / 2)}=\left[\delta_{+1 / 2} B B^{-1}, \psi_{+}^{(+1 / 2)}\right]-\left[\partial_{+} B B^{-1}, D^{(+1 / 2)}\right] .
$$

and

$$
\begin{aligned}
& \delta_{-1 / 2} \psi_{-}^{(-1 / 2)}=-\left[B^{-1} \delta_{-1 / 2} B, \psi_{-}^{(-1 / 2)}\right]-\left[B^{-1} \partial_{-} B, D^{(-1 / 2)}\right], \\
& B^{-1} \delta_{-1 / 2} B=\left[D^{(-1 / 2)}, \psi^{(+1 / 2)}\right], \quad \delta_{-1 / 2} \psi_{+}^{(+1 / 2)}=\left[E_{+}^{(+1)}, B D^{(-1 / 2)} B^{-1}\right] .
\end{aligned}
$$

The physical degrees of freedom are parametrized by the image part $\mathcal{M}$. To guarantee that the variations of the fields remain in $\mathcal{M}$ we have to check that the kernel components of the above transformations vanishes, i.e.

$$
\left[\delta_{+1 / 2} B B^{-1}, \psi_{+}^{(+1 / 2)}\right] \in \mathcal{K}=0, \quad\left[B^{-1} \delta_{-1 / 2} B, \psi_{-}^{(-1 / 2)}\right] \in \mathcal{K}=0
$$

We will see below in the examples that $Q_{ \pm}^{(0)}=0$ imply (19) as a consequence of the absence of the even graded $(2 n, n \in \mathbb{Z})$ part of the bosonic kernel $\mathcal{K}_{B}$ in the mKdV hierarchy. These conditions turn the lowest odd flows $\delta_{ \pm 1 / 2}$ local.

The Lax operators (15), (16) generating the odd flows (17), (18) are related to the rigid 2D supersymmetry transformations of the type

$$
N=\left(N_{+}, N_{-}\right) \text {, }
$$

where $N_{ \pm}=\operatorname{dim} \mathcal{K}_{F}^{( \pm 1 / 2)}$. As the map $D^{( \pm 1 / 2)} \rightarrow \delta_{ \pm 1 / 2}$ obeys

$$
\left[\delta_{ \pm 1 / 2}, \delta_{ \pm 1 / 2}^{\prime}\right](*)=\partial_{\mp\left[D^{ \pm 1 / 2}, D^{\prime \pm 1 / 2}\right]}(*) \sim \partial_{ \pm}(*), \quad\left[\delta_{+1 / 2}, \delta_{-1 / 2}\right](*)=0,
$$

we see that two fermionic transformations close into derivatives, which is by definition a supersymmetry. This is the case provided $\frac{1}{2}\left\{F^{( \pm 1 / 2)}, F^{( \pm 1 / 2)}\right\} \sim E_{ \pm}^{( \pm 1)}$ for $F^{( \pm 1 / 2)} \in \mathcal{K}_{F}^{( \pm 1 / 2)}$, which is significant for the supersymmetric structure of the models, see for instance [18]. For simplicity, we take constant elements $E_{ \pm}^{( \pm 1)}$ which are dual $\left(E_{+}^{(+1)}\right)^{*}=E_{-}^{(-1)}$, giving rise to isomorphic subspaces $\mathcal{K}_{F}^{(+1 / 2)} \simeq \mathcal{K}_{F}^{(-1 / 2)}$ and to $N_{+}=N_{-}$in consistency with the pairing induced by $\left\langle K_{i}, K_{j}\right\rangle \sim \delta_{i+j}$. Note that the non-Abelian odd flows close into the isospectral even flows, as expected, and that the central and gradation fields do not transform under $\delta_{ \pm 1 / 2}$ then, they are not truly degrees of freedom of the model.

\subsection{Recursion operators and higher odd flows}

In computing the explicit expressions for odd Lax operators using (13) generating higher degree fermionic flows we realize that this is considerably more involved than the $\pm 1 / 2$ cases. Instead of that, we use the dressing map $\mathcal{K} \rightarrow \delta_{\mathcal{K}}$ from the kernel algebra to the flow algebra in order to introduce recursion operators. From the relations

$$
\left[\delta_{K^{( \pm 1)}}, \delta_{F^{( \pm 1 / 2)}}\right](*)=\delta_{\left[K^{( \pm 1)}, F^{( \pm 1 / 2)}\right]}(*)=\delta_{F^{( \pm 3 / 2)}}(*),
$$

we infer the following behavior

$$
\begin{aligned}
& \delta_{F^{( \pm n \pm 1 / 2)}}(*)=\operatorname{ad}_{\delta_{K}^{( \pm 1)}}^{n}\left(\delta_{F^{( \pm 1 / 2)}}\right)(*)=\left(\mathcal{R}^{ \pm 1}\right)^{n}\left(\delta_{F^{( \pm 1 / 2)}}\right)(*), \\
& \mathcal{R}^{ \pm 1}(*)=\operatorname{ad}_{\delta_{K^{( \pm 1)}}}(*)=\left[\delta_{K^{( \pm 1)}}, *\right]
\end{aligned}
$$

in terms of the recursion operators $\mathcal{R}^{ \pm 1}$. The aim is not to reproduce the well known supersymmetry transformations but to develop a method to construct systematically all the Higher graded odd symmetry flows in terms of its simplest symmetry structure. However, we have to 
recognize that the use of super pseudo-differential operators and associated scalar Lax operators, seems to be more appropriated for computational purposes.

From this analysis, we have the following two chains of supersymmetry transformations

$$
\begin{aligned}
& \delta_{+1 / 2} \stackrel{\delta_{K^{+}}}{\longrightarrow} \delta_{+3 / 2} \stackrel{\delta_{K^{+}}}{\longrightarrow} \delta_{+5 / 2} \stackrel{\delta_{K^{+}}}{\longrightarrow} \delta_{+7 / 2} \stackrel{\delta_{K^{+}}}{\longrightarrow} \cdots, \\
& \ldots \stackrel{\delta_{K^{-}}}{\leftarrow} \delta_{-7 / 2} \stackrel{\delta_{K^{-}}}{\leftarrow} \delta_{-5 / 2} \stackrel{\delta_{K^{-}}}{\leftarrow} \delta_{-3 / 2} \stackrel{\delta_{K^{-}}}{\leftarrow} \delta_{-1 / 2},
\end{aligned}
$$

where the ones corresponding to $\delta_{ \pm 1 / 2}$ are considered as starting points. The variations $\delta_{K^{ \pm}}$are given by (13) or (14). For example, for a degree +1 element $K^{(+1)}$ we have from (13) that

$$
\begin{aligned}
& \delta_{K^{(+1)}} \psi_{+}^{(+1 / 2)}=-\left[E_{+}^{(+1)},\left.\left(\Theta K^{(+1)} \Theta^{-1}\right)_{-1 / 2}\right|_{\mathcal{M}}\right], \\
& \delta_{K^{(+1)}}\left(\partial_{+} B B^{-1}\right)=+\left[E_{+}^{(+1)},\left(\Theta K^{(+1)} \Theta^{-1}\right)_{-1}\right]+\left[\psi_{+}^{(+1 / 2)},\left.\left(\Theta K^{(+1)} \Theta^{-1}\right)_{-1 / 2}\right|_{\mathcal{K}}\right], \\
& \delta_{K^{(+1)}} \psi_{-}^{(-1 / 2)}=-\left[E_{-}^{(-1)},\left.B^{-1}\left(\Theta K^{(+1)} \Theta^{-1}\right)_{+1 / 2} B\right|_{\mathcal{M}}\right] .
\end{aligned}
$$

The dressing matrix $\Theta$ factorizes as $\Theta=U S$, where $U \in \exp \mathcal{M}$ is local and $S \in \exp \mathcal{K}$ is non-local in the fields [3], splitting the Dressing of the vacuum Lax operators $\left(L_{ \pm}=\Theta L_{ \pm}^{V} \Theta^{-1}\right)$ as a two step process. A $U$ and an $S$ rotation given respectively by

$$
\begin{aligned}
& U^{-1} L_{+} U=\partial_{+}+E_{+}^{(+1)}+K_{+}^{(-)}, \quad U^{-1} L_{-} U=\partial_{-}+K_{-}^{(-)}, \\
& S^{-1}\left(\partial_{+}+E_{+}^{(+1)}+K_{+}^{(-)}\right) S=\partial_{+}+E_{+}^{(+1)}, \quad S^{-1}\left(\partial_{-}+K_{-}^{(-)}\right) S=\partial_{-}+E_{-}^{(-1)},
\end{aligned}
$$

where $K_{ \pm}^{(-)} \in \mathcal{K}$ involve expansions on the negative grades only. The components $\psi^{(i)}, i=$ $-1 / 2,-3 / 2, \ldots$ of $U$ are extracted by projecting (23) along $\mathcal{M}$ and the components $s^{(i)}, i=$ $-1 / 2,-3 / 2, \ldots$ by projecting $(24)$ along $\mathcal{K}$. This allows to compute $(22)$. The higher graded supersymmetry transformations are inevitably non-local because of the presence of the kernel part $S$ appearing in the definition of the transformations $\delta_{K^{( \pm 1)}}$ used to construct them. Thus, the best we can do is to restrict ourselves to a reduced manifold (defined by $Q_{ \pm}^{(0)}=0$ ) in which $\delta_{ \pm 1 / 2}$ are local. From (13) we have

$$
\left[\delta_{K^{(+1)}}, \delta_{-1 / 2}\right](*)=\delta_{\left[K^{(+1)}, D^{(-1 / 2)}\right]_{R}}(*)=0
$$

and we cannot connect $\delta_{-1 / 2}$ and $\delta_{+1 / 2}$ through a $\delta_{K^{(+1)}}$ flow, reflecting the chiral independence of the $\delta_{ \pm 1 / 2}$ transformations as a consequence of the $R$-bracket. This is why in (21) the sectors are treated separately. Although the higher graded odd flows are non-local, their square always give a local even flow. A similar conclusion for this behavior was found in [8] by using superspace formalism.

\subsection{Generalized relativistic-like current identities}

In this section we derive an infinite set of identities associated to the flows generated by $\mathcal{K}_{F}^{( \pm 1 / 2)}$. The word relativistic is used in the sense that each $t_{ \pm n}$ is coupled to its opposite counterpart $t_{\mp n}$.

Proposition 2. The infinite set of fermionic local currents defined by

$$
\begin{array}{ll}
J_{+n}^{(+1 / 2)}=\left\langle D^{(+1 / 2)} \Theta E^{(+n)} \Theta^{-1}\right\rangle, & J_{-n}^{(+1 / 2)}=\left\langle D^{(+1 / 2)} \Pi E^{(-n)} \Pi^{-1}\right\rangle, \\
J_{+n}^{(-1 / 2)}=\left\langle D^{(-1 / 2)} \Theta^{\prime} E^{(+n)} \Theta^{\prime-1}\right\rangle, & J_{-n}^{(-1 / 2)}=\left\langle D^{(-1 / 2)} \Pi^{\prime} E^{(-n)} \Pi^{\prime-1}\right\rangle,
\end{array}
$$

satisfy the following identities

$$
\partial_{+n} J_{-n}^{( \pm 1 / 2)}-\partial_{-n} J_{+n}^{( \pm 1 / 2)}=0 .
$$

The $D^{( \pm 1 / 2)} \in \mathcal{K}_{F}^{( \pm 1 / 2)}$ are the generators of the fermionic kernel. 
Proof. The proof is extremely simple and is based only on the relations (4) and (6). Start with

$$
\begin{aligned}
& \partial_{+n} J_{-m}^{(+1 / 2)}=\left\langle D^{(+1 / 2)}\left[\left(\Theta E^{(+n)} \Theta^{-1}\right)_{\geq 0},\left(\Pi E^{(-m)} \Pi^{-1}\right)_{<0}\right]\right\rangle, \\
& \partial_{-n} J_{+m}^{(+1 / 2)}=\left\langle D^{(+1 / 2)}\left[\left(\Theta E^{(+m)} \Theta^{-1}\right)_{\geq 0},\left(\Pi E^{(-n)} \Pi^{-1}\right)_{<0}\right]\right\rangle
\end{aligned}
$$

to get

$$
\begin{aligned}
\partial_{+n} J_{-n}^{( \pm 1 / 2)}-\partial_{-n} J_{+n}^{( \pm 1 / 2)}= & \left\langle D^{(+1 / 2)}\left[\left(\Theta E^{(+n)} \Theta^{-1}\right)_{\geq 0},\left(\Pi E^{(-m)} \Pi^{-1}\right)_{<0}\right]\right\rangle \\
& -\left\langle D^{(+1 / 2)}\left[\left(\Theta E^{(+m)} \Theta^{-1}\right)_{\geq 0},\left(\Pi E^{(-n)} \Pi^{-1}\right)_{<0}\right]\right\rangle .
\end{aligned}
$$

This sum vanishes for $m=n$. For $J^{(-1 / 2)}$ the proof is analogous.

These identities mixes the two sectors corresponding to positive and negative isospectral times in a relativistic manner. They can be written in a covariant form $\eta^{i j} \frac{\partial}{\partial t_{i}} J_{j}^{( \pm 1 / 2)}=0$ if we define a constant 'metric' $\eta=\eta_{i j} d t_{i} d t_{j}$ for each pair of positive/negative times. However, the interpretation of this higher graded 'light-cone coordinates' deserves further study.

Consider now the lowest isospectral flows $t_{ \pm 1}=-x^{ \pm}$. The current components (25) are given by

$$
\begin{array}{ll}
J_{+}^{(+1 / 2)}=-\left\langle D^{(+1 / 2)}\left[\psi^{(-1 / 2)}, \partial_{+} B B^{-1}\right]\right\rangle, & J_{-}^{(+1 / 2)}=+\left\langle D^{(+1 / 2)} B \psi_{-}^{(-1 / 2)} B^{-1}\right\rangle, \\
J_{+}^{(-1 / 2)}=+\left\langle D^{(-1 / 2)} B^{-1} \psi_{+}^{(+1 / 2)} B\right\rangle, & J_{-}^{(-1 / 2)}=+\left\langle D^{(-1 / 2)}\left[\psi^{(+1 / 2)}, B^{-1} \partial_{-} B\right]\right\rangle .
\end{array}
$$

Then, there are $N=\operatorname{dim} \mathcal{K}_{F}^{( \pm 1 / 2)}$ associated relativistic conservation laws (for each sector) given by $\partial_{+} J_{-}^{( \pm 1 / 2)}-\partial_{-} J_{+}^{( \pm 1 / 2)}=0$. More explicitly, we have

$$
\begin{aligned}
& \partial_{-}\left(\left.\left[\psi^{(-1 / 2)}, \partial_{+} B B^{-1}\right]\right|_{\mathcal{K}}\right)+\partial_{+}\left(\left.B \psi_{-}^{(-1 / 2)} B^{-1}\right|_{\mathcal{K}}\right)=0 \\
& -\partial_{-}\left(\left.B^{-1} \psi_{+}^{(+1 / 2)} B\right|_{\mathcal{K}}\right)+\partial_{+}\left(\left.\left[\psi^{(+1 / 2)}, B^{-1} \partial_{-} B\right]\right|_{\mathcal{K}}\right)=0 .
\end{aligned}
$$

This time, the identities provide supercharge conservation laws due to the fact that the flows $t_{ \pm 1}$ are identified with the light-cone coordinates $x^{ \pm}=\frac{1}{2}\left(x^{0} \pm x^{1}\right)$. It is not clear if the identities associated to the higher flows $t_{ \pm n}, n \geq+1$ provide new conserved quantities because one is not supposed to impose boundary conditions or integrate along these directions. For higher times they are taken as simple identities consequence of the flow relations above.

Now that we have $N=\operatorname{dim} \mathcal{K}_{F}^{( \pm 1 / 2)}$ supercurrents associated to $\mathcal{K}_{F}^{( \pm 1 / 2)}$, let's compute their corresponding supercharges by the Noether procedure in order to check that they really generate the supersymmetry transformations (17) and (18).

\subsection{Supercharges for the SUSY flows $\delta_{ \pm 1 / 2}$}

The action for the affine supersymmetric Toda models was deduced in [15] and it is given by

$$
\begin{aligned}
S_{\text {Toda }}[B, \psi]= & S_{\mathrm{WZNW}}[B]-\frac{k}{4 \pi} \int_{\Sigma}\left\langle\psi_{+}^{(+1 / 2)} \partial_{-} \psi^{(-1 / 2)}+\psi_{-}^{(-1 / 2)} \partial_{+} \psi^{(+1 / 2)}\right\rangle \\
& +\frac{k}{2 \pi} \int_{\Sigma}\left\langle E_{+}^{(+1)} B E_{-}^{(-1)} B^{-1}+\psi_{+}^{(+1 / 2)} B \psi_{-}^{(-1 / 2)} B^{-1}\right\rangle .
\end{aligned}
$$

This corresponds to the situation when we restrict to the sub-superalgebras solving the condition $Q_{ \pm}^{(0)}=0$. In this case the potential ends at the second term providing a Yukawa-type term turning the model integrable and supersymmetric. The light-cone notation used for the flat Minkowski space $\Sigma$ is $x^{ \pm}=\frac{1}{2}\left(x^{0} \pm x^{1}\right), \partial_{ \pm}=\partial_{0} \pm \partial_{1}, \eta_{+-}=\eta_{-+}=2, \eta^{+-}=\eta^{-+}=\frac{1}{2}, \epsilon_{+-}=-\epsilon_{-+}=2$, 
$\epsilon^{-+}=-\epsilon^{+-}=\frac{1}{2}$ corresponding to the metric $\eta_{00}=1, \eta_{11}=-1$ and antisymmetric symbol

$\epsilon_{10}=-\epsilon_{01}=+1$. A coupling constant is introduced by setting $E_{ \pm}^{( \pm 1)} \rightarrow \mu E_{ \pm}^{( \pm 1)}$ and $\psi^{( \pm 1 / 2)} \rightarrow$ $\mu^{-1 / 2} \psi^{( \pm 1 / 2)}$.

An arbitrary variation of the action (28) is given by

$$
\frac{2 \pi}{k} \delta S_{\text {Toda }}=\int_{\Sigma}\left\langle\delta B B^{-1} F_{+-}^{(0)}\right\rangle-\int_{\Sigma}\left\langle\delta \psi^{(+1 / 2)} B^{-1} F_{+-}^{(-1 / 2)} B\right\rangle-\int_{\Sigma}\left\langle\delta \psi^{(-1 / 2)} F_{+-}^{(+1 / 2)}\right\rangle
$$

and the equations of motions are exactly the super Leznov-Saveliev equations, cf. (12) above.

Taking $\delta \rightarrow \delta_{\text {SUSY }}=\delta_{-1 / 2}+\delta_{+1 / 2}$, using (17), (18) and considering $D^{( \pm 1 / 2)}$ as functions of the coordinates $x^{ \pm}$, we have the supersymmetric variation of the action

$$
\begin{aligned}
\frac{2 \pi}{k} \delta_{\text {SUSY }} S_{\text {Toda }}= & \int_{\Sigma}\left\langle\partial_{-} D^{(-1 / 2)}\left(B^{-1} \psi_{+}^{(+1 / 2)} B\right)-\partial_{+} D^{(-1 / 2)}\left[\psi^{(+1 / 2)}, B^{-1} \partial_{-} B\right]\right\rangle \\
& -\int_{\Sigma}\left\langle\partial_{-} D^{(+1 / 2)}\left[\psi^{(-1 / 2)}, \partial_{+} B B^{-1}\right]+\partial_{+} D^{(+1 / 2)}\left(B \psi_{-}^{(-1 / 2)} B^{-1}\right)\right\rangle .
\end{aligned}
$$

This allows to obtain two conservation laws

$$
0=\int_{\Sigma}\left\langle D^{(\mp 1 / 2)}\left(\partial_{-} j_{+}^{( \pm 1 / 2)}+\partial_{+} j_{-}^{( \pm 1 / 2)}\right)\right\rangle
$$

which are exactly the ones derived by using the extended Riemann-Hilbert approach (26) for the lowest flows (27). Then, there are $\operatorname{dim} \mathcal{K}_{F}$ supercurrents and supercharges given by

flow $\delta_{+1 / 2}$ :

$$
\begin{aligned}
& j_{+}^{(-1 / 2)}=\left.\left[\psi^{(-1 / 2)}, \partial_{+} B B^{-1}\right]\right|_{\mathcal{K}}, \quad j_{-}^{(-1 / 2)}=\left.B \psi_{-}^{(-1 / 2)} B^{-1}\right|_{\mathcal{K}} \\
& Q_{+}=\left.\int d x^{1}\left(\left[\psi^{(-1 / 2)}, \partial_{+} B B^{-1}\right]+B \psi_{-}^{(-1 / 2)} B^{-1}\right)\right|_{\mathcal{K}}
\end{aligned}
$$

flow $\delta_{-1 / 2}$ :

$$
\begin{aligned}
& j_{+}^{(+1 / 2)}=-\left.B^{-1} \psi_{+}^{(+1 / 2)} B\right|_{\mathcal{K}}, \quad j_{-}^{(+1 / 2)}=\left.\left[\psi^{(+1 / 2)}, B^{-1} \partial_{-} B\right]\right|_{\mathcal{K}} \\
& Q_{-}=\left.\int d x^{1}\left(\left[\psi^{(+1 / 2)}, B^{-1} \partial_{-} B\right]-B^{-1} \psi_{+}^{(+1 / 2)} B\right)\right|_{\mathcal{K}} .
\end{aligned}
$$

The variation above is the same when (19) are zero or not, this is because all the fields are defined in $\mathcal{M}$ and the kernel part does not affect the variation at all. These two ways of extracting the supercharges show a deep relation between the algebraic dressing formalism and the Hamiltonian reduction giving (28).

Now specialize the construction done above to the simplest toy examples. The supercharges are computed from the general formulas (29) and (30). We want to emphasize that the subsuperalgebras solving the condition $Q_{ \pm}^{(0)}=0$ have no bosonic kernel $\mathcal{K}_{B}$ of degree zero in consistency with the absence of positive even isospectral flows $t_{+2 n}$ in the mKdV hierarchy.

\section{Examples}

These examples show how the superspace notion of supersymmetry can be embedded consistently into the infinite-dimensional flow approach. The usual SUSY transformations corresponds to the flow algebra spanned by the times $\left(t_{-1}, t_{-1 / 2}, t_{+1 / 2}, t_{+1}\right)$. We can have several pairs of odd times $t_{ \pm 1 / 2}$ depending on the dimension of $\mathcal{K}_{F}^{( \pm 1 / 2)}$ as shown above. 


\subsection{The $N=(1,1)$ sinh-Gordon model reloaded}

Take the $\operatorname{sl}(2,1)_{[1]}^{(2)}$ superalgebra (see Appendix A for details). The Lagrangian is

$$
\begin{aligned}
L & =-\frac{k}{2 \pi}\left[\partial_{+} \phi \partial_{-} \phi+\psi_{-} \partial_{+} \psi_{-}+\psi_{+} \partial_{-} \psi_{+}-V\right], \\
V & =2 \mu^{2} \cosh [2 \phi]+4 \mu \psi_{+} \psi_{-} \cosh [\phi]
\end{aligned}
$$

and the equations of motion are

$$
\begin{aligned}
& \partial_{+} \partial_{-} \phi=-2 \mu^{2} \sinh [2 \phi]-2 \mu \psi_{+} \psi_{-} \sinh [\phi], \\
& \partial_{-} \psi_{+}=2 \mu \psi_{-} \cosh [\phi], \quad \partial_{+} \psi_{-}=-2 \mu \psi_{+} \cosh [\phi] .
\end{aligned}
$$
are

With $D^{(+1 / 2)}=\epsilon_{-} F_{2}^{(+1 / 2)}, D^{(-1 / 2)}=\epsilon_{+} F_{1}^{(-1 / 2)}$ and $\psi_{ \pm} \rightarrow \frac{1}{2} \psi_{ \pm}$, the supersymmetry flows

$$
\delta_{ \pm 1 / 2} \phi= \pm \epsilon_{\mp} \psi_{ \pm}, \quad \delta_{ \pm 1 / 2} \psi_{ \pm}=\mp \epsilon_{\mp} \partial_{ \pm} \phi, \quad \delta_{ \pm 1 / 2} \psi_{\mp}=2 \mu \epsilon_{\mp} \sinh [\phi],
$$

where we have used the parametrizations

$$
\begin{aligned}
& B=\exp \left[\phi H_{1}\right], \quad \psi^{(+1 / 2)}=\psi_{-} G_{1}^{(+1 / 2)}, \quad \psi^{(-1 / 2)}=\psi_{+} G_{2}^{(-1 / 2)}, \\
& \psi_{-}^{(-1 / 2)}=2 \psi_{-} G_{2}^{(-1 / 2)}, \quad \psi_{+}^{(+1 / 2)}=-2 \psi_{+} G_{1}^{(+1 / 2)} .
\end{aligned}
$$

We can check (20) by applying the variations twice giving

$$
\left[\delta_{ \pm 1 / 2}, \delta_{ \pm 1 / 2}^{\prime}\right]=2 \epsilon_{\mp} \epsilon_{\mp}^{\prime} \partial_{ \pm}, \quad\left[\delta_{+1 / 2}, \delta_{-1 / 2}\right]=0 .
$$

Then, we have two real supercharges $N=(1,1)$ because of $\operatorname{dim} \mathcal{K}_{F}^{( \pm 1 / 2)}=1$. They are given by

$$
\delta_{ \pm 1 / 2}: \quad Q^{(\mp 1 / 2)}=Q_{ \pm}^{1} F_{1,2}^{(\mp 1 / 2)}, \quad Q_{ \pm}^{1}=\int d x^{1}\left(\psi_{ \pm} \partial_{ \pm} \phi \mp \psi_{\mp} h^{\prime}(\phi)\right)
$$

where $h(\phi)=2 \mu \cosh [\phi]$ and $h^{\prime}(\phi)$ is its functional derivative respect $\phi$.

Now rotate the fermions by the phase $\exp (i \pi / 4)$ in order to write (31) in a more familiar form

$$
\begin{aligned}
& L=-\frac{k}{\pi}\left[\frac{1}{2} \partial_{+} \phi \partial_{-} \phi+\frac{i}{2} \psi_{-} \partial_{+} \psi_{-}+\frac{i}{2} \psi_{+} \partial_{-} \psi_{+}-V\right], \\
& V=\frac{1}{2}\left(h^{\prime}(\phi)\right)^{2}+i h^{\prime \prime}(\phi) \psi_{+} \psi_{-}+\mu^{2},
\end{aligned}
$$

which is known to be invariant under the $N=(1,1)$ superspace transformations for a real bosonic superfield. The area term comes from squaring $h^{\prime}(\phi)=2 \mu \sinh [\phi]$.

Note 1. The Poisson brackets are defined by

$$
\{A, B\}_{\mathrm{PB}}=-(-1)^{\epsilon_{A} \epsilon_{B}}\left(\frac{\partial A}{\partial f} \frac{\partial B}{\partial \pi_{f}}-(-1)^{\epsilon_{A} \epsilon_{B}} \frac{\partial A}{\partial \pi_{f}} \frac{\partial B}{\partial f}\right)
$$

where $\epsilon=1,0$ for bosonic-fermionic quantities and $\pi_{f}=\frac{\partial L}{\partial\left(\partial_{t} f\right)}$. The Dirac bracket is defined by

$$
\{A, B\}_{\mathrm{DB}}=\{A, B\}_{\mathrm{PB}}-\left\{A, \phi_{i}\right\}_{\mathrm{PB}}\left(C^{-1}\right)_{i j}\left\{\phi_{j}, B\right\}_{\mathrm{PB}}
$$

where $C_{i j}=\left\{\phi_{i}, \phi_{i}\right\}_{\mathrm{PB}}$ and $\phi_{i}$ are the second class constraints. 
With the Dirac brackets $\left\{\phi, \partial_{t} \phi\right\}=1,\left\{\psi_{ \pm}, \psi_{ \pm}\right\}=-i$ and $Q_{ \pm}^{1} f=\left\{Q_{ \pm}^{1}, f\right\}$ we have, after replacing $Q_{ \pm}^{1} \rightarrow i Q_{ \pm}^{1}$, the action of the supercharges on the field components

$$
Q_{ \pm}^{1} \phi=-i \psi_{ \pm}, \quad Q_{ \pm}^{1} \psi_{ \pm}=+\partial_{ \pm} \phi, \quad Q_{ \pm}^{1} \psi_{\mp}=\mp h^{\prime}(\phi) .
$$

Finally, the total flow can be written as $\delta_{\mathrm{SUSY}}=-i \epsilon_{-} Q_{+}^{1}+i \epsilon_{+} Q_{-}^{1}$

$$
\delta_{\mathrm{SUSY}} \phi=+i \epsilon_{-} \psi_{+}-i \epsilon_{+} \psi_{-}, \quad \delta_{\mathrm{SUSY}} \psi_{ \pm}=\mp \epsilon_{\mp} \partial_{ \pm} \phi+\epsilon_{ \pm} h^{\prime}(\phi),
$$

which are the ordinary $N=(1,1)$ supersymmetry transformations obtained by using a superspace approach.

Now, construct the $\delta_{+3 / 2}$ transformations starting from $\delta_{+1 / 2}$ by applying $\delta_{K^{(+1)}}$ as shown in (21). From [3] we have

$$
\begin{aligned}
& \delta_{K^{(+1)}} \phi=-\frac{1}{2} \alpha \psi_{+} Q\left(x^{+}\right), \quad \delta_{K^{(+1)}} \psi_{+}=+\frac{1}{2} \alpha\left(\partial_{+} \psi_{+}-\partial_{+} \phi Q\left(x^{+}\right)\right), \\
& \delta_{K^{(+1)}} \psi_{-}=-\frac{1}{2} \alpha\left(\psi_{+} h(\phi)-h^{\prime}(\phi) Q\left(x^{+}\right)\right),
\end{aligned}
$$

where $Q\left(x^{+}\right)=\int^{x^{+}}\left(\psi_{+} \partial_{+} \phi\right)$. From the relation $\left[K_{2}^{(+1)}, F_{2}^{(+1 / 2)}\right]=F_{1}^{(+3 / 2)}$ we have that $\delta_{+3 / 2}=\left[\delta_{K^{(+1)}}, \delta_{+1 / 2}\right]$ and the transformations are given by

$$
\begin{aligned}
& \delta_{+3 / 2} \phi=\epsilon_{-}\left(\frac{1}{2} \partial_{+} \psi_{+}-\partial_{+} \phi Q\left(x^{+}\right)+\frac{1}{2} \psi_{+} H\left(x^{+}\right)\right), \\
& \delta_{+3 / 2} \psi_{+}=\epsilon_{-}\left(\frac{1}{2} \partial_{+}^{2} \phi+\partial_{+} \psi_{+} Q\left(x^{+}\right)-\frac{1}{2} \partial_{+} \phi H\left(x^{+}\right)\right), \\
& \delta_{+3 / 2} \psi_{-}=\epsilon_{-}\left(-\frac{1}{2} \partial_{+} \phi h(\phi)-\psi_{+} Q\left(x^{+}\right) h(\phi)+\frac{1}{2} h^{\prime}(\phi) H\left(x^{+}\right)\right),
\end{aligned}
$$

where $H\left(x^{+}\right)=\int^{x^{+}}\left(\left(\partial_{+} \phi\right)^{2}+\psi_{+} \partial_{+} \psi_{+}\right)$. We also find the variations

$$
\begin{aligned}
& \delta_{+1 / 2} Q\left(x^{+}\right)=-\epsilon_{-} H\left(x^{+}\right), \\
& \delta_{+3 / 2} Q\left(x^{+}\right)=\epsilon_{-}\left(\frac{1}{4}\left(\partial_{+} \phi\right)^{2}-\frac{1}{2} \psi_{+} \partial_{+} \psi_{+}+\partial_{+} \phi \psi_{+} Q\left(x^{+}\right)-\frac{1}{4} H^{2}\left(x^{+}\right)\right), \\
& \delta_{+3 / 2} H\left(x^{+}\right)=\epsilon_{-}\left(\partial_{+} \phi \partial_{+} \psi_{+}-\frac{1}{2} \partial_{+}^{2} \phi \psi_{+}+\frac{1}{2} \partial_{+} \phi \psi_{+} H\left(x^{+}\right)-Q\left(x^{+}\right) \partial_{+} H\left(x^{+}\right)\right) .
\end{aligned}
$$

Applying $\delta_{+3 / 2}$ twice we get a local flow description of the hierarchy for $t_{+1}$ and $t_{+3}$ in terms of the sinh-Gordon variables used to described it in terms of $t_{+1}$ and $t_{-1}$, cf. equation (32)

$$
\begin{aligned}
& 4 \partial_{+3} \phi=\partial_{+}^{3} \phi-2\left(\partial_{+} \phi\right)^{3}-3 \partial_{+} \phi \psi_{+} \partial_{+} \psi_{+}, \quad 4 \partial_{+3} \psi_{+}=\partial_{+}^{3} \psi_{+}-3 \partial_{+} \phi \partial_{+}\left(\partial_{+} \phi \psi_{+}\right), \\
& 4 \partial_{+3} \psi_{-}=\left(2\left(\partial_{+} \phi\right)^{2} \psi_{+}-\partial_{+}^{2} \psi_{+}\right) h(\phi),
\end{aligned}
$$

where $\left[\delta_{+3 / 2}, \delta_{+3 / 2}^{\prime}\right](*)=-2 \epsilon_{1} \epsilon_{2} \partial_{+3}(*)$ in agreement with $\left\{F_{1}^{(+3 / 2)}, F_{1}^{(+3 / 2)}\right\}=-2 E_{+}^{(+3)}$. Introducing $u=\partial_{+} \phi$ (the space variable $x$ is described by $t_{+1}$ ) we recover the super $\mathrm{mKdV}$ equations

$$
\begin{aligned}
& 4 \partial_{+3} u=u^{\prime \prime \prime}-6 u^{2} u^{\prime}-3 \psi_{+}\left(u \psi_{+}^{\prime}\right)^{\prime}, \quad 4 \partial_{+3} \psi_{+}=\psi_{+}^{\prime \prime \prime}-3 u\left(u \psi_{+}\right)^{\prime}, \\
& 4 \partial_{+3} \psi_{-}=\left(2 u^{2} \psi_{+}-\psi_{+}^{\prime \prime}\right) h\left(\partial^{-1} u\right) .
\end{aligned}
$$

The $\psi_{-}$equation is non-local and is remnant from the negative part of the hierarchy. The supersymmetry has to be reduced to the usual $N=(1,0)$ in order to have a local description in terms of the $\mathrm{mKdV}$ variables $u$ and $\psi_{+}$. Note that $Q\left(x^{+}\right)$and $H\left(x^{+}\right)$resembles a piece of the supercharge and a component of the stress tensor. 
Remark 3. The higher grade fermionic transformation are some sort of 'square root' of the isospectral flows, this can be seen also in [8] where Poisson brackets were used (compare with (21)).

The positive and negative parts of the extended $\mathrm{mKdV}$ hierarchy carries exactly the same information when considered separately and to obtain relativistic equations we combine their Lax pairs in different gauges as explained above by general arguments. The potential couples the two sectors and enters through the functional $h(\phi)$. We check this explicitly by considering $\left(t_{+1}, t_{+3}\right),\left(t_{-1}, t_{-3}\right)$ and compute $L_{ \pm 3}$ in order to construct a 1-soliton solution which solves any equation of the hierarchy mixing the four times $\left(t_{+1}, t_{+3}, t_{-1}, t_{-3}\right)$. We keep the name soliton solution for simplicity but strictly speaking we need to have a multi-vacuum theory in order to define the asymptotic boundary values of the field solution.

The algebraic dressing technique (recall equation (4) and (6)) suggest the following forms for the Lax operators

$$
\begin{aligned}
& L_{+3}=\partial_{+3}+D^{(0)}+D^{(+1 / 2)}+D^{(+1)}+D^{(+3 / 2)}+D^{(+2)}+D^{(+5 / 2)}+E^{(+3)}, \\
& L_{-3}=\partial_{-3}-B\left(D^{(-1 / 2)}+D^{(-1)}+D^{(-3 / 2)}+D^{(-2)}+D^{(-5 / 2)}+E^{(-3)}\right) B^{-1} .
\end{aligned}
$$

From $\left[L_{+}, L_{+3}\right]=0$ we get for the positive part of the hierarchy, following [3], the solution

$$
\begin{aligned}
& D^{(+5 / 2)}=\left(-2 \psi_{+}\right) G_{1}^{(+5 / 2)}, \quad D^{(+2)}=\left(-\partial_{+} \phi\right) M_{1}^{(+2)}, \\
& D^{(+3 / 2)}=\left(-\psi_{+} \partial_{+} \phi\right) F_{1}^{(+3 / 2)}+\left(\partial_{+} \psi_{+}\right) G_{2}^{(+3 / 2)}, \\
& D^{(+1)}=\left(-2 \psi_{+} \partial_{+} \psi_{+}\right) K_{1}^{(+1)}+\left(\frac{1}{2}\left(\partial_{+} \phi\right)^{2}+2 \psi_{+} \partial_{+} \psi_{+}\right) K_{2}^{(+1)}+\left(\frac{1}{2} \partial_{+}^{2} \phi\right) M_{2}^{(+1)}, \\
& D^{(+1 / 2)}=-\frac{1}{2}\left(\partial_{+}^{2} \phi \psi_{+}-\partial_{+} \phi \partial_{+} \psi_{+}\right) F_{2}^{(+1 / 2)}+\left(-\frac{1}{2} \partial_{+}^{2} \psi_{+}+\psi_{+}\left(\partial_{+} \phi\right)^{2}\right) G_{1}^{(+1 / 2)}, \\
& D^{(0)}=\left(-\frac{1}{4} \partial_{+}^{3} \phi+\frac{1}{2}\left(\partial_{+} \phi\right)^{3}+3 \partial_{+} \phi \psi_{+} \partial_{+} \psi_{+}\right) M_{1}^{(0)} .
\end{aligned}
$$

The equation of motion are given by the degree zero component and are, as expected, given the first two equations of (34), after taking $\psi_{+} \rightarrow \frac{1}{2} \psi_{+}$. Now, performing a gauge transformation with $B^{-1}$ in order to eliminate the $B$ conjugation on $L_{-3}$ and $L_{-}$we change to

$$
\begin{aligned}
& L_{-}=\partial_{-}+B^{-1} \partial_{-} B-\left(E_{-}^{(-1)}+\psi_{-}^{(-1 / 2)}\right), \\
& L_{-3}=\partial_{-3}+B^{-1} \partial_{-3} B-\left(D^{(-1 / 2)}+D^{(-1)}+D^{(-3 / 2)}+D^{(-2)}+D^{(-5 / 2)}+E^{(-3)}\right)
\end{aligned}
$$

and from $\left[L_{-}, L_{-3}\right]=0$ we get for the negative part of the hierarchy, the solutions

$$
\begin{aligned}
& D^{(-5 / 2)}=\left(2 \psi_{-}\right) G_{2}^{(-5 / 2)}, \quad D^{(-2)}=\left(-\partial_{-} \phi\right) M_{1}^{(-2)} \\
& D^{(-3 / 2)}=\left(\psi_{-} \partial_{-} \phi\right) F_{2}^{(-3 / 2)}+\left(\partial_{-} \psi_{-}\right) G_{1}^{(-3 / 2)} \\
& D^{(-1)}=\left(-2 \psi_{-} \partial_{-} \psi_{-}\right) K_{1}^{(-1)}+\left(\frac{1}{2}\left(\partial_{-} \phi\right)^{2}+2 \psi_{-} \partial_{-} \psi_{-}\right) K_{2}^{(-1)}+\left(-\frac{1}{2} \partial_{-}^{2} \phi\right) M_{2}^{(-1)}, \\
& D^{(-1 / 2)}=-\frac{1}{2}\left(\partial_{-}^{2} \phi \psi_{-}-\partial_{-} \phi \partial_{-} \psi_{-}\right) F_{1}^{(-1 / 2)}+\left(\frac{1}{2} \partial_{-}^{2} \psi_{-}-\left(\partial_{-} \phi\right)^{2} \psi_{-}\right) G_{2}^{(-1 / 2)} .
\end{aligned}
$$

The equations of motion are now

$$
4 \partial_{-3} \phi=\partial_{-}^{3} \phi-2\left(\partial_{-} \phi\right)^{3}-3 \partial_{-} \phi \psi_{-} \partial_{-} \psi_{-}, \quad 4 \partial_{-3} \psi_{-}=\partial_{-}^{3} \psi_{-}-3 \partial_{-} \phi \partial_{-}\left(\partial_{-} \phi \psi_{-}\right)
$$

after taking $\psi_{-} \rightarrow \frac{1}{2} \psi_{-}$. With $v=\partial_{-} \phi$ (the space variable $x$ is described by $t_{-1}$ ) we get

$$
4 \partial_{-3} v=v^{\prime \prime \prime}-6 v^{2} v-3 \psi_{-}\left(v \psi_{-}^{\prime}\right)^{\prime}, \quad 4 \partial_{-3} \psi_{-}=\psi_{-}^{\prime \prime \prime}-3 v\left(v \psi_{-}\right)^{\prime} .
$$


From the solution of the equation (43), i.e. $D^{(0)}=-\partial_{+3} B B^{-1}$ we confirm that $4 \partial_{+3} \phi=$ $\partial_{+}^{3} \phi-2\left(\partial_{+} \phi\right)^{3}-3 \partial_{+} \phi \psi_{+} \partial_{+} \psi_{+}$, after taking $\psi_{+} \rightarrow \frac{1}{2} \psi_{+}$. This is the $N=(0,1)$ mKdV equation with opposite chirality.

The simplest example of a relativistic equation is provided by the sinh-Gordon model which corresponds to the lowest $t_{ \pm 1}$ times. Now compute $\left[L_{+3}, L_{-3}\right]=0$ with $L_{-3}$ in the form (35), i.e. in its original gauge because the two copies are to be taken in different gauges. The generalized relativistic system of equations is given in the Appendix B, equation (44), and is a generalization to the $t_{ \pm 3}$ times. The two sectors of the extended hierarchy are identical, thus we complete the system (34) with the following set of equations

$$
\begin{aligned}
& 4 \partial_{-3} \phi=\partial_{-}^{3} \phi-2\left(\partial_{-} \phi\right)^{3}-3 \partial_{-} \phi \psi_{-} \partial_{-} \psi_{-}, \quad 4 \partial_{-3} \psi_{-}=\partial_{-}^{3} \psi_{-}-3 \partial_{-} \phi \partial_{-}\left(\partial_{-} \phi \psi_{-}\right), \\
& 4 \partial_{-3} \psi_{+}=\left(2\left(\partial_{-} \phi\right)^{2} \psi_{-}-\partial_{-}^{2} \psi_{-}\right) h(\phi)
\end{aligned}
$$

and due to this symmetric behavior we can easily read off the $\delta_{-3 / 2}$ transformations directly from (33) simply by replacing all + sub-indexes by - sub-indexes.

Now consider for simplicity the bosonic limit of the generalized equations (44). It is given by

$$
\begin{aligned}
\partial_{+3} \partial_{-3} \phi= & -2 \sinh [2 \phi]+\frac{1}{2}\left(\left(\partial_{+} \phi\right)^{2}\left(\partial_{-} \phi\right)^{2}+\partial_{+}^{2} \phi \partial_{-}^{2} \phi\right) \sinh [2 \phi] \\
& -\frac{1}{2}\left(\partial_{+}^{2} \phi\left(\partial_{-} \phi\right)^{2}+\left(\partial_{+} \phi\right)^{2} \partial_{-}^{2} \phi\right) \cosh [2 \phi], \\
\partial_{ \pm 3}\left(\partial_{\mp} \phi\right)= & \left(\partial_{ \pm} \phi\right)^{2} \sinh [2 \phi]-\partial_{ \pm}^{2} \phi \cosh [2 \phi], \\
\partial_{ \pm 3}\left(\partial_{\mp} \phi\right)^{2}= & 2 \partial_{\mp} \phi\left(\partial_{ \pm} \phi\right)^{2} \sinh [2 \phi]-2 \partial_{\mp} \phi \partial_{ \pm}^{2} \phi \cosh [2 \phi], \\
\partial_{ \pm 3}\left(\partial_{\mp}^{2} \phi\right)= & 4 \partial_{ \pm} \phi+2 \partial_{\mp} \phi\left(\partial_{ \pm} \phi\right)^{2} \cosh [2 \phi]-2 \partial_{\mp} \phi \partial_{ \pm}^{2} \phi \sinh [2 \phi]
\end{aligned}
$$

and describe the behavior of the ' $\phi$-descendants' $\partial_{ \pm} \phi,\left(\partial_{ \pm} \phi\right)^{2}$ and $\partial_{ \pm}^{2} \phi$ in terms of opposite $t_{\mp 3}$ times. The equation (37) can be obtained alternatively by using the basic non-linear relations $\partial_{ \pm 3} \phi=\frac{1}{4} \partial_{ \pm}^{3} \phi-\frac{1}{2}\left(\partial_{ \pm} \phi\right)^{3}$, and the lowest relativistic equation $\partial_{+} \partial_{-} \phi=-2 \sinh [2 \phi]$. The whole set of equations associated to the times $\left(t_{-3}, t_{-1}, t_{+1}, t_{+3}\right)$ is completed by the equations extracted from the relations $\left[L_{+}, L_{-3}\right]=0$ and $\left[L_{-}, L_{+3}\right]=0$. They are given by the sinh-Gordon equation itself, the equations on the third line of (37) and

$$
\begin{aligned}
& 4 \partial_{\mp} \phi=\partial_{ \pm}\left(\partial_{\mp} \phi\right)^{2} \sinh [2 \phi]-\partial_{ \pm} \partial_{\mp}^{2} \phi \cosh [2 \phi], \\
& 0=\partial_{ \pm} \partial_{\mp}^{2} \phi \sinh [2 \phi]-\partial_{ \pm}\left(\partial_{\mp} \phi\right)^{2} \cosh [2 \phi] .
\end{aligned}
$$

Now take $K^{ \pm(n)}=E^{( \pm n)}$ in (13) and conjugate them with the grading operator $Q$ as follows

$$
E^{\prime( \pm n)}=\exp (\alpha Q) E^{( \pm n)} \exp (-\alpha Q)=\exp ( \pm n) E^{( \pm n)}=\Lambda^{ \pm n} E^{( \pm n)},
$$

where $\Lambda=\exp (\alpha)$. The equations are invariant under these rescalings and the Lorentz transformations, i.e. $x^{\prime \pm}=\Lambda^{ \pm 1} x^{ \pm}$can be generalized to the whole set of flows by taking $t_{ \pm n}^{\prime}=\Lambda^{ \pm n} t_{ \pm n}$.

To find the 1-soliton solution of the equation (37) we use the dressing method which is another application of the formalism given above in Section 2.1, see also [16] for computational details. The four vacuum Lax operators involved are

$$
L_{ \pm}=\partial_{ \pm} \pm E_{ \pm}^{( \pm 1)}, \quad L_{ \pm 3}=\partial_{ \pm 3} \pm E^{( \pm 3)}
$$

and the zero curvature conditions imply they are pure gauge $A_{i}^{V}=T_{0}^{-1} \partial_{i} T_{0}$. Hence we have as usual

$$
A_{+}^{V}=E_{+}^{(+1)}-x^{-} C, \quad A_{-}^{V}=-E_{-}^{(-1)}, \quad A_{+3}^{V}=E^{(+3)}-3 t_{-3} C, \quad A_{-3}^{V}=-E^{(-3)},
$$




$$
T_{0}=\exp \left(t_{+3} E^{(+3)}+x^{+} E_{+}^{(+1)}\right) \exp \left(-x^{-} E_{-}^{(-1)}-t_{-3} E^{(-3)}\right),
$$

where we have used $\left[E^{(m)}, E^{(n)}\right]=\frac{1}{2}(m-n) \delta_{m+n, 0} C$ for $(m, n)$ odd integers. The dressing of a vacuum Lax connections $A_{i}^{V}$ is the gauge transformation $A_{i}=\left(\Theta_{ \pm}\right)^{-1} A_{i}^{V} \Theta_{ \pm}+\left(\Theta_{ \pm}\right)^{-1} \partial_{i} \Theta_{ \pm}$, satisfying $\Theta_{-} \Theta_{+}^{-1}=T_{0}^{-1} g T_{0}$, where $g$ is an arbitrary constant group element. Assuming that $\Theta_{-}^{-1}=e^{p(-1)} e^{p(-2)} \cdots, \Theta_{+}^{-1}=e^{q(0)} e^{q(1)} e^{q(2)} \cdots$, where $p(-i)$ and $q(i)$ are linear combinations of grade $(-i)$ and $(+i)$ respectively, the zero grade component of $A_{i}$ leads to the solution

$$
e^{q(0)}=B^{-1} e^{-\nu C}, \quad B=\exp (\phi H) .
$$

From this we have

$$
\left\langle\lambda^{\prime}\left|B^{-1}\right| \lambda\right\rangle e^{-\nu}=\left\langle\lambda^{\prime}\left|T_{0}^{-1} g T_{0}\right| \lambda\right\rangle,
$$

where $|\lambda\rangle$ and $\left\langle\lambda^{\prime}\right|$ are annihilated by the grade $(-i)$ and $(i)$ generators. Taking the highest weight states $\left|\lambda_{i}\right\rangle, i=0,1$ of $\operatorname{sl}(2)^{(1)}$ we get the following tau-functions

$$
\tau_{1}=e^{-\phi-\nu}=\left\langle\lambda_{1}\left|T_{0}^{-1} g T_{0}\right| \lambda_{1}\right\rangle, \quad \tau_{0}=e^{-\nu}=\left\langle\lambda_{0}\left|T_{0}^{-1} g T_{0}\right| \lambda_{0}\right\rangle .
$$

The so called solitonic specialization corresponds to the situation when $g$ is given by the exponential of an eigenvalue $F(z), z \in \mathbb{C}$ of the operator ad $E^{(k)}$. In this case we have

$$
\left[E^{(n)}, F(z)\right]=-2 z^{n} F(z),
$$

where $F(z)$ is the vertex operator

$$
F(z)=\sum_{n=-\infty}^{+\infty}\left[\left(M_{1}^{(2 n)}-\frac{1}{2} \delta_{n, 0} C\right) z^{-2 n}+M_{2}^{(2 n+1)} z^{-2 n-1}\right] .
$$

With the following result

$$
T_{0}^{-1} g T_{0}=\exp \{\rho(z) F(z)\}, \quad \rho(\gamma)=\exp 2\left\{-t_{-3} z^{-3}-x^{-} z^{-1}+x^{+} z+t_{+3} z^{3}\right\},
$$

we get the 1-soliton solution depending on the first four times of the hierarchy

$$
\phi\left(t_{-3}, x^{-}, x^{+}, t_{+3}\right)=-\log \left(\frac{\tau_{1}}{\tau_{0}}\right),
$$

where $\tau_{1}=1+\frac{b}{2} \rho(z), \tau_{0}=1-\frac{b}{2} \rho(z)$ and $b=$ const. The field (40) is a simultaneous solution of the bosonic limits (with $\mu=1$ ) of the equations (32), (34), (36) and the whole set of equations (37) and (38). The interesting point is that the second and third terms of the RHS of (37) cancel each other and the field $\phi$ has to obey

$$
\partial_{+3} \partial_{-3} \phi=-2 \sinh [2 \phi],
$$

which is the case. Then, the solution (40) behaves under $t_{ \pm 3}$ like a sinh-Gordon soliton solution but with a cubic rapidity. For higher grade times the extension in (39) is direct. At this point we can notice that each chirality of the extended hierarchy is attached separately to the poles $z=+\infty$ (positive flows) and $z=0$ (negative flows) of the Lax pair with spectral parameter $z$, see for instance [7]. This is exactly the pole structure entering the definition of the sinh-Gordon Lax pair.

We end by giving the fermionic currents for the times $t_{ \pm 3}$ and $D^{(+1 / 2)}$. After a lengthly but straightforward computation, the equation (26) becomes $\partial_{+3} J_{-3}^{(+1 / 2)}+\partial_{-3} J_{+3}^{(+1 / 2)}=0$ with the current components

$$
\begin{aligned}
-\frac{1}{8} j_{+3}^{(+1 / 2)} & =\partial_{-} \phi \partial_{-} \psi_{-}-\psi_{-} \partial_{-}^{2} \phi \\
-\frac{1}{8} j_{-3}^{(+1 / 2)} & =-2 \psi_{+} \sinh [\phi]-\left(\frac{1}{2} \partial_{+}^{2} \psi_{+} \partial_{+} \phi-\psi_{+}\left(\partial_{+} \phi\right)^{3}-\frac{1}{2} \partial_{+} \psi_{+} \partial_{+}^{2} \phi\right) \cosh [\phi] .
\end{aligned}
$$




\subsection{The $N=(2,2)$ Landau-Ginzburg Toda model}

Take the $\operatorname{psl}(2,2)_{[3]}^{(2)}$ superalgebra (see Appendix A for details). The Lagrangian is given by

$$
\begin{aligned}
L= & -\frac{k}{2 \pi}\left[\partial_{+} \phi_{1} \partial_{-} \phi_{1}+\partial_{+} \phi_{3} \partial_{-} \phi_{3}+\psi_{1} \partial_{+} \psi_{1}+\psi_{3} \partial_{+} \psi_{3}+\bar{\psi}_{2} \partial_{-} \bar{\psi}_{2}+\bar{\psi}_{4} \partial_{-} \bar{\psi}_{4}-V\right], \\
V= & 2 \cosh \left[2 \phi_{1}\right]-2 \cos \left[2 \phi_{3}\right]-4\left(\psi_{1} \bar{\psi}_{2}+\psi_{3} \bar{\psi}_{4}\right) \cosh \left[\phi_{1}\right] \cos \left[\phi_{3}\right] \\
& +4\left(\psi_{1} \bar{\psi}_{4}-\psi_{3} \bar{\psi}_{2}\right) \sinh \left[\phi_{1}\right] \sin \left[\phi_{3}\right] .
\end{aligned}
$$

Taking $D^{(+1 / 2)}=D_{1}^{(+1 / 2)}+D_{2}^{(+1 / 2)}$ with $D_{1}^{(+1 / 2)}=\epsilon_{1} F_{1}^{(+1 / 2)}, \quad D_{2}^{(+1 / 2)}=\epsilon_{2} F_{4}^{(+1 / 2)}$ and $D^{(-1 / 2)}=D_{1}^{(-1 / 2)}+D_{2}^{(-1 / 2)}$ with $D_{1}^{(-1 / 2)}=\bar{\epsilon}_{1} F_{2}^{(-1 / 2)}, D_{2}^{(-1 / 2)}=\bar{\epsilon}_{2} F_{3}^{(-1 / 2)}$, the supersymmetry transformations (17) and (18) with $\psi_{i}, \bar{\psi}_{i} \rightarrow \frac{1}{2} \psi_{i}, \frac{1}{2} \bar{\psi}_{i}$ are

$$
\begin{aligned}
& \delta_{+1 / 2} \phi_{1}=+2\left(\epsilon_{1} \bar{\psi}_{4}-\epsilon_{2} \bar{\psi}_{2}\right), \quad \delta_{+1 / 2} \phi_{3}=-2\left(\epsilon_{2} \bar{\psi}_{4}+\epsilon_{1} \bar{\psi}_{2}\right), \\
& \delta_{+1 / 2} \bar{\psi}_{2}=+\left(\epsilon_{2} \partial_{+} \phi_{1}+\epsilon_{1} \partial_{+} \phi_{3}\right), \quad \delta_{+1 / 2} \bar{\psi}_{4}=-\left(\epsilon_{1} \partial_{+} \phi_{1}-\epsilon_{2} \partial_{+} \phi_{3}\right), \\
& \delta_{+1 / 2} \psi_{1}=-2 \epsilon_{1} \cosh \left[\phi_{1}\right] \sin \left[\phi_{3}\right]-2 \epsilon_{2} \sinh \left[\phi_{1}\right] \cos \left[\phi_{3}\right], \\
& \delta_{+1 / 2} \psi_{3}=+2 \epsilon_{1} \sinh \left[\phi_{1}\right] \cos \left[\phi_{3}\right]-2 \epsilon_{2} \cosh \left[\phi_{1}\right] \sin \left[\phi_{3}\right], \\
& \delta_{-1 / 2} \phi_{1}=+2\left(\bar{\epsilon}_{1} \psi_{3}-\bar{\epsilon}_{2} \psi_{1}\right), \quad \delta_{-1 / 2} \phi_{3}=+2\left(\bar{\epsilon}_{1} \psi_{1}+\bar{\epsilon}_{2} \psi_{3}\right), \\
& \delta_{-1 / 2} \psi_{1}=+\left(\bar{\epsilon}_{2} \partial_{-} \phi_{1}-\bar{\epsilon}_{1} \partial_{-} \phi_{3}\right), \quad \delta_{-1 / 2} \psi_{3}=-\left(\bar{\epsilon}_{1} \partial_{-} \phi_{1}+\bar{\epsilon}_{2} \partial_{-} \phi_{3}\right), \\
& \delta_{-1 / 2} \bar{\psi}_{2}=-2 \bar{\epsilon}_{1} \cosh \left[\phi_{1}\right] \sin \left[\phi_{3}\right]+2 \bar{\epsilon}_{2} \sinh \left[\phi_{1}\right] \cos \left[\phi_{3}\right], \\
& \delta_{-1 / 2} \bar{\psi}_{4}=-2 \bar{\epsilon}_{1} \sinh \left[\phi_{1}\right] \cos \left[\phi_{3}\right]-2 \bar{\epsilon}_{2} \cosh \left[\phi_{1}\right] \sin \left[\phi_{3}\right] .
\end{aligned}
$$

We can check (20) by applying (41) twice giving

$$
\begin{aligned}
& {\left[\delta_{+1 / 2}, \delta_{+1 / 2}^{\prime}\right]=+4\left(\epsilon_{1} \epsilon_{1}^{\prime}+\epsilon_{2} \epsilon_{2}^{\prime}\right) \partial_{+}, \quad\left[\delta_{-1 / 2}, \delta_{-1 / 2}^{\prime}\right]=+4\left(\bar{\epsilon}_{1} \bar{\epsilon}_{1}^{\prime}+\bar{\epsilon}_{2} \bar{\epsilon}_{2}^{\prime}\right) \partial_{-},} \\
& {\left[\delta_{+1 / 2}, \delta_{-1 / 2}\right]=0 .}
\end{aligned}
$$

We have four real supercharges $N=(2,2)$ because $\operatorname{dim} \mathcal{K}_{F}^{( \pm 1 / 2)}=2$. They are extracted from (29) and (30) and are given by

flow $\delta_{+1 / 2}$ :

$$
\begin{aligned}
& Q^{(-1 / 2)}=Q_{+}^{1} F_{2}^{(-1 / 2)}+Q_{+}^{2} F_{3}^{(-1 / 2)}, \\
& Q_{+}^{1}=\int d x^{1}\left(\bar{\psi}_{2} \partial_{+} \phi_{3}-\bar{\psi}_{4} \partial_{+} \phi_{1}-2 \psi_{1} \cosh \left[\phi_{1}\right] \sin \left[\phi_{3}\right]+2 \psi_{3} \sinh \left[\phi_{1}\right] \cos \left[\phi_{3}\right]\right), \\
& Q_{+}^{2}=\int d x^{1}\left(\bar{\psi}_{4} \partial_{+} \phi_{3}+\bar{\psi}_{2} \partial_{+} \phi_{1}-2 \psi_{1} \sinh \left[\phi_{1}\right] \cos \left[\phi_{3}\right]-2 \psi_{3} \cosh \left[\phi_{1}\right] \sin \left[\phi_{3}\right]\right),
\end{aligned}
$$

flow $\delta_{-1 / 2}$ :

$$
\begin{aligned}
& Q^{(+1 / 2)}=Q_{-}^{1} F_{1}^{(+1 / 2)}+Q_{-}^{2} F_{4}^{(+1 / 2)}, \\
& Q_{-}^{1}=\int d x^{1}\left(\psi_{1} \partial_{-} \phi_{3}+\psi_{3} \partial_{-} \phi_{1}+2 \bar{\psi}_{2} \cosh \left[\phi_{1}\right] \sin \left[\phi_{3}\right]+2 \bar{\psi}_{4} \sinh \left[\phi_{1}\right] \cos \left[\phi_{3}\right]\right), \\
& Q_{-}^{2}=\int d x^{1}\left(\psi_{3} \partial_{-} \phi_{3}-\psi_{1} \partial_{-} \phi_{1}-2 \bar{\psi}_{2} \sinh \left[\phi_{1}\right] \cos \left[\phi_{3}\right]+2 \bar{\psi}_{4} \cosh \left[\phi_{1}\right] \sin \left[\phi_{3}\right]\right) .
\end{aligned}
$$

Now introduce the complex fields

$$
\psi_{-}=\psi_{1}+i \psi_{3}, \quad \psi_{+}=-\bar{\psi}_{2}+i \bar{\psi}_{4}, \quad \phi=\phi_{3}+i \phi_{1}
$$


and the superpotential $W(\phi)=2 \mu \cos \phi$ in order to write the lagrangian in a more familiar form. Then, we have

$$
\begin{aligned}
& L=-\frac{k}{2 \pi}\left[\partial_{+} \phi \partial_{-} \phi^{*}+\psi_{-}^{*} \partial_{+} \psi_{-}+\psi_{+}^{*} \partial_{-} \psi_{+}-V\right], \\
& V=\left|W^{\prime}(\phi)\right|^{2}-\left[W^{\prime \prime}(\phi) \psi_{-} \psi_{+}+W^{* \prime \prime}\left(\phi^{*}\right) \psi_{-}^{*} \psi_{+}^{*}\right],
\end{aligned}
$$

where $\left|W^{\prime}(\phi)\right|^{2}=2 \mu^{2} \cosh \left[2 \phi_{1}\right]-2 \mu^{2} \cos \left[2 \phi_{3}\right]$. This Lagrangian is invariant under the common $N=(2,2)$ superspace transformations for a complex chiral bosonic superfield. In terms of the new complex fields we have for (41) with $\epsilon_{-}=-\left(\epsilon_{1}+i \epsilon_{2}\right)$ and $\epsilon_{+}=\bar{\epsilon}_{1}-i \bar{\epsilon}_{2}$ that

$$
\begin{array}{ll}
\delta_{+1 / 2} \phi=-2 \epsilon_{-} \psi_{+}, & \delta_{-1 / 2} \phi=+2 \epsilon_{+} \psi_{-}, \\
\delta_{+1 / 2} \psi_{+}=+\epsilon_{-}^{*} \partial_{+} \phi, & \delta_{-1 / 2} \psi_{+}=-i \epsilon_{+} W^{\prime *}\left(\phi^{*}\right), \\
\delta_{+1 / 2} \psi_{-}=-i \epsilon_{-} W^{\prime *}\left(\phi^{*}\right), & \delta_{-1 / 2} \psi_{-}=-\epsilon_{+}^{*} \partial_{-} \phi
\end{array}
$$

plus their complex conjugates. Define now the following complex combinations of the supercharges $\frac{1}{2} Q_{ \pm}=\mp Q_{ \pm}^{1}+i Q_{ \pm}^{2}$

$$
Q_{ \pm}=2 \int d x^{1}\left(\psi_{ \pm} \partial_{ \pm} \phi^{*} \mp i \psi_{\mp}^{*} W^{\prime *}\left(\phi^{*}\right)\right), \quad \bar{Q}_{ \pm}=2 \int d x^{1}\left(\psi_{ \pm}^{*} \partial_{ \pm} \phi \pm i \psi_{\mp} W^{\prime}(\phi)\right) .
$$

Now, with the Dirac brackets $\left\{\phi, \partial_{t} \phi^{*}\right\}=\left\{\phi^{*}, \partial_{t} \phi\right\}=+1$ and $\left\{\psi_{+}, \psi_{+}^{*}\right\}=\left\{\psi_{-}, \psi_{-}^{*}\right\}=+1 / 2$ we have with $Q_{ \pm} f=\left\{Q_{ \pm}, f\right\}$

$$
\begin{array}{ll}
Q_{+} \phi=-2 \psi_{+}, & \bar{Q}_{+} \phi=0, \\
Q_{+} \psi_{+}=0, & \bar{Q}_{+} \psi_{+}=\partial_{+} \phi, \\
Q_{+} \psi_{-}=-i W^{*}\left(\phi^{*}\right), & \bar{Q}_{+} \psi_{-}=0, \\
Q_{-} \phi=-2 \psi_{-}, & \bar{Q}_{-} \phi=0, \\
Q_{-} \psi_{+}=i W^{\prime *}\left(\phi^{*}\right), & \bar{Q}_{-} \psi_{+}=0, \\
Q_{-} \psi_{-}=0, & \bar{Q}_{-} \psi_{-}=\partial_{-} \phi
\end{array}
$$

plus their complex conjugates.

Finally, the total variation becomes $\delta_{\mathrm{SUSY}}=\epsilon_{-} Q_{+}-\epsilon_{+} Q_{-}+\epsilon_{-}^{*} \bar{Q}_{+}-\epsilon_{+}^{*} \bar{Q}_{-}$with

$$
\begin{array}{ll}
\delta_{\mathrm{SUSY}} \phi=+2 \epsilon_{+} \psi_{-}-2 \epsilon_{-} \psi_{+}, & \delta_{\mathrm{SUSY}} \phi^{*}=+2 \epsilon_{+}^{*} \psi_{-}^{*}-2 \epsilon_{-}^{*} \psi_{+}^{*}, \\
\delta_{\mathrm{SUSY}} \psi_{+}=+\epsilon_{-}^{*} \partial_{+} \phi-i \epsilon_{+} W^{\prime *}\left(\phi^{*}\right), & \delta_{\mathrm{SUSY}} \psi_{+}^{*}=+\epsilon_{-} \partial_{+} \phi^{*}+i \epsilon_{+}^{*} W^{\prime}(\phi), \\
\delta_{\mathrm{SUSY}} \psi_{-}=-\epsilon_{+}^{*} \partial_{-} \phi-i \epsilon_{-} W^{\prime *}\left(\phi^{*}\right), & \delta_{\mathrm{SUSY}} \psi_{-}^{*}=-\epsilon_{+} \partial_{-} \phi^{*}+i \epsilon_{-}^{*} W^{\prime}(\phi),
\end{array}
$$

which are the usual $N=(2,2)$ supersymmetry transformations obtained by using a superspace approach. As in the case of the $N=(1,1)$ model, we expect the existence of higher non-local fermionic flows for this model.

Remark 4. The action (42) is a Landau-Ginzburg model on a flat non-compact trivial CalabiYau manifold $X$, i.e. $X=\mathbb{C}$. As is well known this model is $B$-twistable. It would be interesting to see the relation of its chiral ring and the chiral ring of a topologically twisted version of a superstring on $\operatorname{AdS}^{2} \times S^{2}$ where the action (42) is extracted as a Pohlmeyer reduction [17]. This would be in principle, a simple way to test up to what point the Pohlmeyer reduction can be understood as an equivalence of quantum field theories, at least at the level of ground states and by eliminating conformal anomaly issues. It would be also interesting to trace back (if possible) the role played by the extended conformal symmetries of $\mathcal{W}$-type in terms of sigma model variables. 
Remark 5. In the relativistic sector of the AKNS hierarchy which is associated to the Homogeneous gradation, the Toda potential has a symmetry, i.e. $\mathcal{K}_{B}^{(0)} \neq \varnothing$. In this case further reduction of the model can be performed, eliminating the flat directions. This is done by coupling to a quadratic gauge field $A$ and is equivalent to the introduction of singular metrics defining the non-Abelian Toda models also known as singular Toda models. The minimal coupling of the fermions with the gauge field gives two terms proportional to $Q_{ \pm}^{(0)}$. After integration of $A$, the action will have a potential term which is quartic in the fermions, roughly of the form

$$
V=\left\langle Q_{+}^{(0)} B Q_{-}^{(0)} B^{-1}\right\rangle \sim R(B) \psi_{+}^{1} \psi_{+}^{2} \psi_{-}^{1} \psi_{-}^{2},
$$

which is possibly related to the curvature $R$ of the background metric. This hierarchy is relevant from the point of view of Pohlmeyer reductions where reductions of non-linear sigma models inside this hierarchy are quite common [17].

\section{Concluding remarks}

By coupling two identical supersymmetric integrable hierarchies we have shown that the usual notion of superspace/supersymmetry is embedded and alternatively described by the symmetry algebra spanned by the subset of flows $\left(t_{-1}, t_{-1 / 2}, t_{+1 / 2}, t_{+1}\right) \subset\left(t_{ \pm 1 / 2}, t_{ \pm 1}, t_{ \pm 3 / 2}, \ldots\right)$. We have given the explicit form of the supercharges generating these extended $(N, N)$ supersymmetry flows and also shown that the higher grade fermionic flows are inevitably non-local on both chiral sectors. In particular, when the $\left(t_{-1}, t_{-1 / 2}, t_{+1 / 2}, t_{+1}\right)$ flows are supplemented by the algebraic conditions $Q_{ \pm}^{(0)}=0$, the integrable model is restricted to a reduced group manifold spanned by the invariant subalgebra of a reductive automorphism $\tau_{\text {red }}$, see Appendix A for an explicit example. We do not supersymmetrize the fields, i.e. the 'angles' that parametrize the group elements $\widehat{G}$ as usually done in the literature. The reduction by $\tau_{\text {red }}$ should be, in the general case, a natural extension to superalgebras of the automorphism used to define the bosonic affine (Abelian) Toda models, see [2] for an example applied to the Lie algebra $\widehat{\mathfrak{g}}=\operatorname{sl}(m+1, \mathbb{C})$. The reduction provides a well defined connection between the dressing elements and the physical degrees of freedom cf. equation (11), as well as the right number of terms in the potential appearing in the action functional by truncating it at the second term, thus the natural affine superalgebras involved in the supersymmetric affine (Abelian) Toda models are the twisted ones.

What remains to be done in general terms is a formal proof of the statement that the introduction of $\tau_{\text {red }}$ is responsible for the locality $\left(Q_{ \pm}^{(0)}=0\right)$ of the lowest supersymmetry flows $\left(\delta_{ \pm 1 / 2}\right)$. This is equivalent to an explicit construction of a reductive automorphism $\tau_{\text {red }}$ in which

the invariant subalgebra $\widehat{\mathfrak{g}}_{\text {red }}$ has no bosonic kernel of grade zero, i.e. $\mathcal{K}_{B}^{(0)}=\varnothing$. Such $\tau_{\text {red }}$ will define in principle, all supersymmetric affine Abelian Toda models attached to the mKdV hierarchy (in the A series) and it would be interesting to construct it also for other series of affine superalgebras. The next step is to introduce a super tau-function formulation which we expect to be a natural generalization of the one introduced in [21] for the bosonic affine Toda models, where an infinite number of conserved charges $Q_{n}^{ \pm}$were written in terms of the boundary values $B$ of a single tau function $\tau$, in the form $\left.Q_{n}^{ \pm} \sim \partial_{ \pm n} \log \tau\right|_{B}$. As we are using a fermionic version of the Toda models coupled to matter fields constructed in [13], it is possible that one has to consider a 'matrix' of tau functions $\tau_{m n}$ when solving the whole system (12). In this case, the interpretation of the single $\tau$ function as a classical limit of the partition function of some quantum integrable system will change or will have to be modified in an appropriate way. The point is that quantization can be done, in principle, by quantizing $\tau_{m n}$, i.e. by using a quantum group of dressing transformations. This obviously deserves a separate study. 
A potential application for our supersymmetric affine Toda models is related to the Pohlmeyer reduction of supersymmetric sigma models. In the present construction we needed to impose the conditions $Q_{ \pm}^{(0)}=0$ in order to have $\mathcal{K}_{B}^{(0)}=\varnothing$. This means that the corresponding supersymmetric reduced models belong to the $\mathrm{mKdV}$ hierarchy and that no gauge symmetries are involved, thus having Toda models of Abelian type (see equation (42) for an example). To apply these results in the most general situation we need to find a way of coupling two supersymmetric AKNS hierarchies in which $\mathcal{K}_{B}^{(0)} \neq \varnothing$, i.e. the reduced models will have gauge symmetries, thus being Toda models of non-Abelian type. We expect to deduce the action functional for the supersymmetric non-Abelian affine Toda models similar to the one constructed in [17, equation (6.49)] in the particular case of the reduction of the $\mathrm{AdS}_{5} \times S^{5}$ superstring coset sigma model. We also expect to deduce the supersymmetry transformations by treating them as fermionic symmetry flows in the AKNS hierarchy in the same way as it was done for the action (28) and (17), (18) in the mKdV hierarchy. It is worth to compare our supersymmetry transformations with the ones proposed in [17, equations (7.21), (7.22)]. We also comment that our Lax pair and action functional naturally includes a spectral parameter and that it is also conformal invariant as a consequence of the two-loop nature of the affine algebras used. The study of the 'off shell' supersymmetric AKNS hierarchy and its symmetries is already under investigation [23].

\section{A Used superalgebras solving $Q_{ \pm}^{(0)}=0$}

We consider the reductive automorphism $\tau_{\text {red }}$ for $\operatorname{sl}(2,1)^{(1)}$ only [3]. It is defined by

$$
\begin{array}{ll}
\tau_{\text {red }}\left(E_{\alpha}^{(n)}\right)=-(-1)^{q\left(E_{\alpha}^{(n)}\right)} E_{-\alpha}^{\left(n+\eta_{E_{\alpha}}\right)} & \text { for } \alpha \in \text { bosonic root, } \\
\tau_{\text {red }}\left(E_{\alpha}^{(n)}\right)=i(-1)^{q\left(E_{\alpha}^{(n)}\right)} E_{-\alpha}^{\left(n+\eta_{E_{\alpha}}\right)} & \text { for } \alpha \in \text { fermionic root, }
\end{array}
$$

where $q\left(E_{\alpha}^{(n)}\right)$ is the grade of $E_{\alpha}^{(n)}$, i.e. $\left[Q, E_{\alpha}^{(n)}\right]=q\left(E_{\alpha}^{(n)}\right) E_{\alpha}^{(n)}, \eta_{E_{\alpha}}$ is defined by $\left[\alpha \cdot H, E_{\alpha}\right]=$ $\eta_{E_{\alpha}} E_{\alpha}$ and $\alpha$ are the roots of the underlying finite-dimensional Lie superalgebra.

Invariance under $\tau_{\text {red }}$ define a twisted superalgebra $\operatorname{sl}(2,1)^{(2)} \subset \operatorname{sl}(2,1)^{(1)}$. This twisted superalgebra solve the conditions $Q_{ \pm}^{(0)}=0$. In [15], two sub-superalgebras of $\operatorname{sl}(2,1)^{(1)}$ solving the conditions $Q_{ \pm}^{(0)}=0$ where found by another method. The first algebra solution was denoted by $\operatorname{sl}(2,1)_{[1]}^{(2)}$ and coincides with the subalgebra invariant under $\tau_{\text {red }}$ above. The second algebra solution was denoted by $\operatorname{sl}(2,1)_{[2]}^{(2)}$. These two subalgebras gives rise to integrable models of sinh-Gordon and sine-Gordon type which are related by analytic continuation in the fields. Similarly, in the case of $\operatorname{psl}(2,2)^{(1)}$, four sub-superalgebras solving the conditions $Q_{ \pm}^{(0)}=0$ were found. they were denoted as $\operatorname{psl}(2,2)_{[i]}^{(2)}, i=1,2,3,4$ and give rise to integrable field theories coupling models of (sinh-sinh), (sine,sine), (sinh,sine) and (sine, sinh) Gordon type respectively, all of them related by analytic continuation. The subscript $[i]$ is just a label used to denote a particular subalgebra solving the locallity conditions $Q_{ \pm}^{(0)}=0$. The reason of using these subalgebras is to turn local the lowest supersymmetry flows. The explicit difference, for the case $\operatorname{sl}(2,1)^{(1)}$ (non-local) and $\operatorname{sl}(2,1)_{[1]}^{(2)}$ (local) was worked out in detail in [3].

In this paper we consider, for the sake of simplicitly and with the aim of not being repetitive, only the superalgebras $\operatorname{sl}(2,1)_{[1]}^{(2)}$ and $\operatorname{psl}(2,2)_{[3]}^{(2)}$ whose definitions are as follows

$$
\begin{aligned}
& \operatorname{sl}(2,1)_{[1]}^{(2)}: \\
& Q=2 d+\frac{1}{2} H_{1}, \quad E_{ \pm}^{( \pm 1)}=\lambda_{2} \cdot H^{( \pm 1 / 2)}-\left(E_{ \pm \alpha_{1}}^{(0)}+E_{\mp \alpha_{1}}^{( \pm 1)}\right) \quad \text { and } \\
& \mathcal{K}_{B}=\left\{K_{1}^{(2 n+1)}=\lambda_{2} \cdot H^{(n+1 / 2)}, K_{2}^{(2 n+1)}=E_{\alpha_{1}}^{(n)}+E_{-\alpha_{1}}^{(n+1)}\right\}
\end{aligned}
$$




$$
\begin{aligned}
& \mathcal{M}_{B}=\left\{M_{1}^{(2 n)}=H_{1}^{(n)}, M_{2}^{(2 n+1)}=E_{\alpha_{1}}^{(n)}-E_{-\alpha_{1}}^{(n+1)}\right\}, \\
& \mathcal{K}_{F}=\left\{\begin{array}{c}
F_{1}^{(2 n+3 / 2)}=\left(E_{\alpha_{2}}^{(n+1)}+E_{\alpha_{1}+\alpha_{2}}^{(n+1 / 2)}\right)+\left(E_{-\alpha_{2}}^{(n+1 / 2)}+E_{-\alpha_{1}-\alpha_{2}}^{(n+1)}\right), \\
F_{2}^{(2 n+1 / 2)}=\left(E_{\alpha_{2}}^{(n+1 / 2)}+E_{\alpha_{1}+\alpha_{2}}^{(n)}-\left(E_{-\alpha_{2}}^{(n)}+E_{-\alpha_{1}-\alpha_{2}}^{(n+1 / 2)}\right)\right.
\end{array}\right\}, \\
& \mathcal{M}_{F}=\left\{\begin{array}{c}
G_{1}^{(2 n+1 / 2)}=\left(E_{\alpha_{2}}^{(n+1 / 2)}-E_{\alpha_{1}+\alpha_{2}}^{(n)}\right)+\left(E_{-\alpha_{2}}^{(n)}-E_{-\alpha_{1}-\alpha_{2}}^{(n+1 / 2)}\right), \\
G_{2}^{(2 n+3 / 2)}=\left(E_{\alpha_{2}}^{(n+1)}-E_{\alpha_{1}+\alpha_{2}}^{(n+1 / 2)}\right)-\left(E_{-\alpha_{2}}^{(n+1 / 2)}-E_{-\alpha_{1}-\alpha_{2}}^{(n+1)}\right)
\end{array}\right\} .
\end{aligned}
$$

$$
\begin{aligned}
& Q=d+\frac{1}{2}\left(H_{1}+H_{3}\right), \quad E_{ \pm}^{( \pm 1)}=\left(E_{ \pm \alpha_{1}}^{(0)}+E_{\mp \alpha_{1}}^{( \pm 1)}\right)+\left(E_{ \pm \alpha_{3}}^{(0)}+E_{\mp \alpha_{3}}^{( \pm 1)}\right)+I \quad \text { and } \\
& \mathcal{K}_{B}=\left\{K_{1}^{(2 n+1)}=I^{(2 n+1)}, K_{2}^{(2 n+1)}=E_{\alpha_{1}}^{(2 n)}+E_{-\alpha_{1}}^{(2 n+2)}, K_{3}^{(2 n+1)}=E_{\alpha_{3}}^{(2 n+2)}+E_{-\alpha_{3}}^{(2 n)}\right\} \text {, } \\
& \mathcal{M}_{B}=\left\{\begin{array}{c}
M_{1}^{(2 n)}=H_{1}^{(2 n)}, M_{2}^{(2 n+1)}=E_{\alpha_{1}}^{(2 n)}-E_{-\alpha_{1}}^{(2 n+2)}, \\
M_{3}^{(2 n+1)}=H_{3}^{(2 n+1)}, M_{4}^{(2 n)}=E_{\alpha_{3}}^{(2 n+1)}-E_{-\alpha_{3}}^{(2 n-1)}
\end{array}\right\}, \\
& \mathcal{K}_{F}=\left\{\begin{array}{c}
F_{1}^{(2 n+1 / 2)}=\left(E_{\alpha_{2}}^{(2 n+1 / 2)}+E_{\alpha_{1}+\alpha_{2}+\alpha_{3}}^{(2 n+1 / 2)}+\left(E_{\alpha_{1}+\alpha_{2}}^{(2 n-1 / 2)}+E_{\alpha_{2}+\alpha_{3}}^{(2 n+3 / 2)}\right)+\right. \\
+\left(E_{-\alpha_{2}}^{(2 n+1 / 2)}+E_{-\alpha_{1}-\alpha_{2}-\alpha_{3}}^{(2 n+1 / 2)}+\left(E_{-\alpha_{1}-\alpha_{2}}^{(2 n+3 / 2)}+E_{-\alpha_{2}-\alpha_{3}}^{(2 n-1 / 2)},\right.\right. \\
F_{2}^{(2 n+3 / 2)}=\left(E_{\alpha_{2}}^{(2 n+3 / 2)}+E_{\alpha_{1}+\alpha_{2}+\alpha_{3}}^{(2 n+3 / 2)}+\left(E_{\alpha_{1}+\alpha_{2}}^{(2 n+1 / 2)}+E_{\alpha_{2}+\alpha_{3}}^{(2 n+5 / 2)}\right)-\right. \\
-\left(E_{-\alpha_{2}}^{(2 n+3 / 2)}+E_{-\alpha_{1}-\alpha_{2}-\alpha_{3}}^{(2 n+3 / 2)}-\left(E_{-\alpha_{1}-\alpha_{2}}^{(2 n+5 / 2)}+E_{-\alpha_{2}-\alpha_{3}}^{(2 n+1 / 2)}\right),\right. \\
F_{3}^{(2 n+3 / 2)}=\left(E_{\alpha_{2}}^{(2 n+3 / 2)}+E_{\alpha_{1}+\alpha_{2}+\alpha_{3}}^{(2 n+3 / 2)}-\left(E_{\alpha_{1}+\alpha_{2}}^{(2 n+1 / 2)}+E_{\alpha_{2}+\alpha_{3}}^{(2 n+5 / 2)}\right)+\right. \\
+\left(E_{-\alpha_{2}}^{(2 n+3 / 2)}+E_{-\alpha_{1}-\alpha_{2}-\alpha_{3}}^{(2 n+3 / 2)}-\left(E_{-\alpha_{1}-\alpha_{2}}^{(2 n+5 / 2)}+E_{-\alpha_{2}-\alpha_{3}}^{(2 n+1 / 2)}\right),\right. \\
F_{4}^{(2 n+1 / 2)}=-\left(E_{\alpha_{2}}^{(2 n+1 / 2)}+E_{\alpha_{1}+\alpha_{2}+\alpha_{3}}^{(2 n+1 / 2)}\right)+\left(E_{\alpha_{1}+\alpha_{2}}^{(2 n-1 / 2)}+E_{\alpha_{2}+\alpha_{3}}^{(2 n+3 / 2)}\right)+ \\
+\left(E_{-\alpha_{2}}^{(2 n+1 / 2)}+E_{-\alpha_{1}-\alpha_{2}-\alpha_{3}}^{(2 n+1 / 2)}-\left(E_{-\alpha_{1}-\alpha_{2}}^{(2 n+3 / 2)}+E_{-\alpha_{2}-\alpha_{3}}^{(2 n-1 / 2)}\right)\right.
\end{array}\right\}, \\
& \mathcal{M}_{F}=\left\{\begin{array}{c}
G_{1}^{(2 n+1 / 2)}=\left(E_{\alpha_{1}+\alpha_{2}}^{(2 n-1 / 2)}-E_{\alpha_{2}+\alpha_{3}}^{(2 n+3 / 2)}\right)+\left(E_{\alpha_{2}}^{(2 n+1 / 2)}-E_{\alpha_{1}+\alpha_{2}+\alpha_{3}}^{(2 n+1 / 2)}\right) \\
+\left(E_{-\alpha_{1}-\alpha_{2}}^{(2 n+3 / 2)}-E_{-\alpha_{2}-\alpha_{3}}^{(2 n-1 / 2)}+\left(E_{-\alpha_{2}}^{(2 n+1 / 2)}-E_{-\alpha_{1}-\alpha_{2}-\alpha_{3}}^{(2 n+1 / 2),}\right.\right. \\
G_{2}^{(2 n+3 / 2)}=\left(E_{\alpha_{1}+\alpha_{2}}^{(2 n+1 / 2)}-E_{\alpha_{2}+\alpha_{3}}^{(2 n+5 / 2)}\right)+\left(E_{\alpha_{2}}^{(2 n+3 / 2)}-E_{\alpha_{1}+\alpha_{2}+\alpha_{3}}^{(2 n+3 / 2)}\right)- \\
-\left(E_{-\alpha_{1}-\alpha_{2}}^{(2 n+5 / 2)}-E_{-\alpha_{2}-\alpha_{3}}^{(2 n+1 / 2)}-\left(E_{-\alpha_{2}}^{(2 n+3 / 2)}-E_{-\alpha_{1}-\alpha_{2}-\alpha_{3}}^{(2 n+3 / 2)},\right.\right. \\
G_{3}^{(2 n+3 / 2)}=\left(E_{\alpha_{1}+\alpha_{2}}^{(2 n+1 / 2)}-E_{\alpha_{2}+\alpha_{3}}^{(2 n+5 / 2)}\right)-\left(E_{\alpha_{2}}^{(2 n+3 / 2)}-E_{\alpha_{1}+\alpha_{2}+\alpha_{3}}^{(2 n+3 / 2)}\right)+ \\
+\left(E_{-\alpha_{1}-\alpha_{2}}^{(2 n+5 / 2)}-E_{-\alpha_{2}-\alpha_{3}}^{(2 n+1 / 2)}-\left(E_{-\alpha_{2}}^{(2 n+3 / 2)}-E_{\left.-\alpha_{1}-\alpha_{2}-\alpha_{3}\right),}^{(2 n+3 / 2)}\right.\right. \\
G_{4}^{(2 n+1 / 2)}=-\left(E_{\alpha_{1}+\alpha_{2}}^{(2 n-1 / 2)}-E_{\alpha_{2}+\alpha_{3}}^{(2 n+1 / 2)}\right)+\left(E_{\alpha_{2}}^{(2 n+1 / 2)}-E_{\alpha_{1}+\alpha_{2}+\alpha_{3}}^{(2 n+1 / 2)}\right)+ \\
+\left(E_{-\alpha_{1}-\alpha_{2}}^{(2 n+3 / 2)}-E_{-\alpha_{2}-\alpha_{3}}^{(2 n-1 / 2)}-\left(E_{-\alpha_{2}}^{(2 n+1 / 2)}-E_{-\alpha_{1}-\alpha_{2}-\alpha_{3}}^{(2 n+1 / 2)}\right)\right.
\end{array}\right\} .
\end{aligned}
$$

\section{B Relativistic equations for $t_{ \pm 3}$}

The decomposition of the zero curvature equation $\left[L_{+3}, L_{-3}\right]=0$ on the different graded subspaces reads

$$
\begin{aligned}
& \partial_{ \pm 3} D^{(\mp 5 / 2)}= \pm\left[E^{(\mp 3)}, B^{\mp 1} D^{( \pm 1 / 2)} B^{ \pm 1}\right], \\
& \partial_{ \pm 3} D^{(\mp 2)}= \pm\left[D^{(\mp 5 / 2)}, B^{\mp 1} D^{( \pm 1 / 2)} B^{ \pm 1}\right] \pm\left[E^{(\mp 3)}, B^{\mp 1} D^{( \pm 1)} B^{ \pm 1}\right] \\
& \partial_{ \pm 3} D^{(\mp 3 / 2)}= \pm\left[D^{(\mp 2)}, B^{\mp 1} D^{( \pm 1 / 2)} B^{ \pm 1}\right] \pm\left[D^{(\mp 5 / 2)}, B^{\mp 1} D^{( \pm 1)} B^{ \pm 1}\right]
\end{aligned}
$$




$$
\begin{aligned}
& \pm\left[E^{(\mp 3)}, B^{\mp 1} D^{( \pm 3 / 2)} B^{ \pm 1}\right], \\
& \partial_{ \pm 3} D^{(\mp 1)}= \pm {\left[D^{(\mp 3 / 2)}, B^{\mp 1} D^{( \pm 1 / 2)} B^{ \pm 1}\right] \pm\left[D^{(\mp 2)}, B^{\mp 1} D^{( \pm 1)} B^{ \pm 1}\right] } \\
& \pm {\left[D^{(\mp 5 / 2)}, B^{\mp 1} D^{( \pm 3 / 2)} B^{ \pm 1}\right] \pm\left[E^{(\mp 3)}, B^{\mp 1} D^{( \pm 2)} B^{ \pm 1}\right] } \\
& \partial_{ \pm 3} D^{(\mp 1 / 2)}= \pm\left[D^{(\mp 1)}, B^{\mp 1} D^{( \pm 1 / 2)} B^{ \pm 1}\right] \pm\left[D^{(\mp 3 / 2)}, B^{\mp 1} D^{( \pm 1)} B^{ \pm 1}\right] \\
& \pm\left[D^{(\mp 2)}, B^{\mp 1} D^{( \pm 3 / 2)} B^{ \pm 1}\right] \pm\left[D^{(\mp 5 / 2)}, B^{\mp 1} D^{( \pm 2)} B^{ \pm 1}\right] \\
& \pm\left[E^{(\mp 3)}, B^{\mp 1} D^{( \pm 5 / 2)} B^{ \pm 1}\right], \\
& \partial_{-3}\left(\partial_{+3} B B^{-1}\right)= {\left[D^{(+1 / 2)}, B D^{(-1 / 2)} B^{-1}\right]+\left[D^{(+1)}, B D^{(-1)} B^{-1}\right] } \\
&+\left[D^{(+3 / 2)}, B D^{(-3 / 2)} B^{-1}\right]+\left[D^{(+2)}, B D^{(-2)} B^{-1}\right] \\
&+\left[D^{(+5 / 2)}, B D^{(-5 / 2)} B^{-1}\right]+\left[E^{(+3)}, B E^{(-3)} B^{-1}\right],
\end{aligned}
$$

where we have replaced the solution $D^{(0)}=-\partial_{+3} B B^{-1}$ for the degree -3 equation

$$
0=\left[D^{(0)}+\partial_{+3} B B^{-1}, B E^{(+3)} B^{-1}\right] .
$$

For the $\operatorname{sl}(2,1)_{[1]}^{(2)}$ algebra set

$$
\begin{aligned}
& D^{(+5 / 2)}=\psi_{+}^{1} G_{1}^{(+5 / 2)}, \quad D^{(-5 / 2)}=\psi_{-}^{1} G_{2}^{(-5 / 2)}, \\
& D^{(+2)}=\phi_{+}^{1} M_{1}^{(+2)}, \quad D^{(-2)}=\phi_{-}^{1} M_{1}^{(-2)}, \\
& D^{(+3 / 2)}=\psi_{+}^{2} F_{1}^{(+3 / 2)}+\psi_{+}^{3} G_{2}^{(+3 / 2)}, \quad D^{(-3 / 2)}=\psi_{-}^{2} F_{2}^{(-3 / 2)}+\psi_{-}^{3} G_{1}^{(-3 / 2)} \text {, } \\
& D^{(+1)}=\phi_{+}^{2} K_{1}^{(+1)}+\phi_{+}^{3} K_{2}^{(+1)}+\phi_{+}^{4} M_{2}^{(+1)}, \quad D^{(-1)}=\phi_{-}^{2} K_{1}^{(-1)}+\phi_{-}^{3} K_{2}^{(-1)}+\phi_{-}^{4} M_{2}^{(-1)} \text {, } \\
& D^{(+1 / 2)}=\psi_{+}^{4} F_{2}^{(+1 / 2)}+\psi_{+}^{5} G_{1}^{(+1 / 2)}, \quad D^{(-1 / 2)}=\psi_{-}^{4} F_{1}^{(-1 / 2)}+\psi_{-}^{5} G_{2}^{(-1 / 2)} \text {, } \\
& D^{(0)}=\phi_{+}^{5} M_{1}^{(0)}
\end{aligned}
$$

then, the above equations of motion are

$$
\begin{aligned}
\partial_{ \pm 3} \psi_{\mp}^{1}= & 2 \psi_{ \pm}^{4} \sinh [\phi] \pm 2 \psi_{ \pm}^{5} \cosh [\phi], \\
\partial_{ \pm 3} \phi_{\mp}^{1}= & 2 \psi_{\mp}^{1} \psi_{ \pm}^{4} \cosh [\phi] \pm 2 \psi_{\mp}^{1} \psi_{ \pm}^{5} \sinh [\phi]-2 \phi_{ \pm}^{3} \sinh [2 \phi] \pm 2 \phi_{ \pm}^{4} \cosh [2 \phi], \\
\partial_{ \pm 3} \psi_{\mp}^{2}= & -\phi_{\mp}^{1} \psi_{ \pm}^{4} \sinh [\phi] \mp \phi_{\mp}^{1} \psi_{ \pm}^{5} \cosh [\phi]+\psi_{\mp}^{1} \phi_{ \pm}^{3} \sinh [2 \phi] \mp \psi_{\mp}^{1} \phi_{ \pm}^{4} \cosh [2 \phi], \\
\partial_{ \pm 3} \psi_{\mp}^{3}= & \mp\left(\phi_{\mp}^{1} \psi_{ \pm}^{4}-2 \psi_{ \pm}^{3}\right) \cosh [\phi]-\left(\phi_{\mp}^{1} \psi_{ \pm}^{5}-2 \psi_{ \pm}^{2}\right) \sinh [\phi] \pm \phi_{ \pm}^{3} \psi_{\mp}^{1} \cosh [2 \phi] \\
& -\psi_{\mp}^{1} \phi_{ \pm}^{4} \sinh [2 \phi] \pm \psi_{\mp}^{1} \phi_{ \pm}^{2}, \\
\partial_{ \pm 3} \phi_{\mp}^{2}= & 2\left(-\psi_{\mp}^{2} \psi_{ \pm}^{4}+\psi_{\mp}^{3} \psi_{ \pm}^{5}-\psi_{\mp}^{1} \psi_{ \pm}^{3}\right) \cosh [\phi] \pm 2\left(-\psi_{\mp}^{2} \psi_{ \pm}^{5}+\psi_{\mp}^{3} \psi_{ \pm}^{4}-\psi_{\mp}^{1} \psi_{ \pm}^{2}\right) \sinh [\phi], \\
\partial_{ \pm 3} \phi_{\mp}^{3}= & 2\left(-\psi_{\mp}^{2} \psi_{ \pm}^{4}-\psi_{\mp}^{3} \psi_{ \pm}^{5}+\psi_{\mp}^{1} \psi_{ \pm}^{3}\right) \cosh [\phi] \mp 2\left(\psi_{\mp}^{2} \psi_{ \pm}^{5}+\psi_{\mp}^{3} \psi_{ \pm}^{4}-\psi_{\mp}^{1} \psi_{ \pm}^{2}\right) \sinh [\phi] \\
& -2 \phi_{\mp}^{1} \phi_{ \pm}^{3} \sinh [2 \phi] \pm 2 \phi_{\mp}^{1} \phi_{ \pm}^{4} \cosh [2 \phi] \\
\partial_{ \pm 3} \phi_{\mp}^{4}= & \pm 2\left(\psi_{\mp}^{2} \psi_{ \pm}^{4}+\psi_{\mp}^{3} \psi_{ \pm}^{5}-\psi_{\mp}^{1} \psi_{ \pm}^{3}\right) \sinh [\phi]+2\left(\psi_{\mp}^{2} \psi_{ \pm}^{5}+\psi_{\mp}^{3} \psi_{ \pm}^{4}-\psi_{\mp}^{1} \psi_{ \pm}^{2}\right) \cosh [\phi] \\
& \pm 2 \phi_{\mp}^{1} \phi_{ \pm}^{3} \cosh [2 \phi]-2 \phi_{\mp}^{1} \phi_{ \pm}^{4} \sinh [2 \phi] \pm 2 \phi_{ \pm}^{1} \\
\partial_{ \pm 3} \psi_{\mp}^{4}= & \left(\phi_{\mp}^{2} \psi_{ \pm}^{4}-\phi_{\mp}^{3} \psi_{ \pm}^{4}-\phi_{\mp}^{4} \psi_{ \pm}^{5}+\phi_{\mp}^{1} \psi_{ \pm}^{3}\right) \cosh [\phi] \\
& +\left(\phi_{\mp}^{4} \psi_{ \pm}^{4}-\phi_{\mp}^{2} \psi_{ \pm}^{5}+\phi_{\mp}^{3} \psi_{ \pm}^{5}-\phi_{\mp}^{1} \psi_{ \pm}^{2}\right) \sinh [\phi] \\
& \mp\left(\phi_{ \pm}^{3} \psi_{\mp}^{2}+\phi_{ \pm}^{4} \psi_{\mp}^{3}\right) \cosh [2 \phi]+\left(\psi_{\mp}^{2} \phi_{ \pm}^{4}+\psi_{\mp}^{3} \phi_{ \pm}^{3}\right) \sinh [2 \phi] \pm \psi_{\mp}^{1} \phi_{ \pm}^{1} \pm \psi_{\mp}^{2} \phi_{ \pm}^{2}, \\
\partial_{ \pm 3} \psi_{\mp}^{5}= & \mp\left(\phi_{\mp}^{4} \psi_{+}^{4}+\phi_{\mp}^{2} \psi_{ \pm}^{5}+\phi_{\mp}^{3} \psi_{ \pm}^{5}+\phi_{\mp}^{1} \psi_{ \pm}^{2}-2 \psi_{ \pm}^{1}\right) \cosh [\phi] \\
& -\left(\phi_{\mp}^{2} \psi_{ \pm}^{4}+\phi_{\mp}^{3} \psi_{ \pm}^{4}+\phi_{\mp}^{4} \psi_{ \pm}^{5}+\phi_{\mp}^{1} \psi_{ \pm}^{3}\right) \sinh [\phi]-\left(\psi_{\mp}^{2} \phi_{ \pm}^{3}+\psi_{\mp}^{3} \phi_{ \pm}^{4}\right) \sinh [2 \phi] \\
& \pm\left(\psi_{\mp}^{2} \phi_{ \pm}^{4}+\psi_{\mp}^{3} \phi_{ \pm}^{3}\right) \cosh [2 \phi] \pm \psi_{\mp}^{3} \phi_{ \pm}^{2},
\end{aligned}
$$




$$
\begin{aligned}
\partial_{+3} \partial_{-3} \phi= & 2\left(\psi_{+}^{4} \psi_{-}^{4}-\psi_{+}^{5} \psi_{-}^{5}-\psi_{+}^{2} \psi_{-}^{2}+\psi_{+}^{3} \psi_{-}^{3}-\psi_{+}^{1} \psi_{-}^{1}\right) \sinh [\phi] \\
& +2\left(\psi_{+}^{4} \psi_{-}^{5}+\psi_{-}^{4} \psi_{+}^{5}-\psi_{+}^{2} \psi_{-}^{3}-\psi_{-}^{2} \psi_{+}^{3}\right) \cosh [\phi] \\
& -2\left(1-\phi_{+}^{3} \phi_{-}^{3}+\phi_{+}^{4} \phi_{-}^{4}\right) \sinh [2 \phi]-2\left(\phi_{+}^{3} \phi_{-}^{4}-\phi_{-}^{3} \phi_{+}^{4}\right) \cosh [2 \phi] .
\end{aligned}
$$

The generalized equations of motion are found by replacing above the following solutions

$$
\begin{aligned}
& \psi_{ \pm}^{1}=\mp 2 \psi_{ \pm}, \quad \psi_{ \pm}^{2}=\mp \psi_{ \pm} \partial_{ \pm} \phi, \quad \psi_{ \pm}^{3}=\partial_{ \pm} \psi_{ \pm}, \quad \psi_{ \pm}^{4}=-\frac{1}{2}\left(\psi_{ \pm} \partial_{ \pm}^{2} \phi-\partial_{ \pm} \phi \partial_{ \pm} \psi_{ \pm}\right), \\
& \psi_{ \pm}^{5}=\mp \frac{1}{2} \partial_{ \pm}^{2} \psi_{ \pm} \pm \psi_{ \pm}\left(\partial_{ \pm} \phi\right)^{2}, \quad \phi_{ \pm}^{1}=-\partial_{ \pm} \phi, \quad \phi_{ \pm}^{2}=-2 \psi_{ \pm} \partial_{ \pm} \psi_{ \pm}, \\
& \phi_{ \pm}^{3}=\frac{1}{2}\left(\partial_{ \pm} \phi\right)^{2}+2 \psi_{ \pm} \partial_{ \pm} \psi_{ \pm}, \quad \phi_{ \pm}^{4}= \pm \frac{1}{2} \partial_{ \pm}^{2} \phi, \\
& \phi_{+}^{5}=-\frac{1}{4} \partial_{+}^{3} \phi+\frac{1}{2}\left(\partial_{+} \phi\right)^{3}+3 \partial_{+} \phi \psi_{+} \partial_{+} \psi_{+} .
\end{aligned}
$$

\section{Acknowledgements}

The author thanks FAPESP and CNPq for partial financial support, J.F. Gomes for comments and the referees for useful suggestions. The author also thanks Alexis Roa and Suzana Moreira for reading the manuscript.

\section{References}

[1] Aratyn H., Gomes J.F., de Castro G.M., Silka M.B., Zimerman A.H., Supersymmetry for integrable hierarchies on loop superalgebras, J. Phys. A: Math. Gen. 38 (2005), 9341-9357, hep-th/0508008.

[2] Aratyn H., Gomes J.F., Zimerman A.H., Integrable hierarchy for multidimensional Toda equations and topological-anti-topological fusion, J. Geom. Phys. 46 (2003), 21-47, Erratum, J. Geom. Phys. 46 (2003), 201, hep-th/0107056.

[3] Aratyn H., Gomes J.F., Zimerman A.H., Supersymmetry and the KdV equations for integrable hierarchies with a half-integer gradation, Nuclear Phys. B 676 (2004), 537-571, hep-th/0309099.

[4] Aratyn H., Gomes J.F., Zimerman A.H., Nisimov E., Pacheva S., Symmetry flows, conservation laws and dressing approach to the integrable models, in Integrable Hierarchies and Modern Physical Theories (Chicago, 2000), NATO Sci. Ser. II Math. Phys. Chem., Vol. 18, Kluwer Acad. Publ., Dordrecht, 2001, 243-275, nlin.SI/0012042.

[5] Au G., Spence B., Hamiltonian reduction and supersymmetric Toda models, Modern Phys. Lett. A 10 (1995), 2157-2168, hep-th/9505026.

[6] Babelon O., Bernard D., Dressing symmetries, Comm. Math. Phys. 149 (1992), 279-306, hep-th/9111036.

[7] Babelon O., Bernard D., Talon M., Introduction to classical integrable systems, Cambridge Monographs on Mathematical Physics, Cambridge University Press, Cambridge, 2003.

[8] Dargis P., Mathieu P., Nonlocal conservation laws for supersymmetric KdV equations, Phys. Lett. A 176 (1993), 67-74, hep-th/9301080.

[9] Delduc F., Gallot L., Supersymmetric Drinfeld-Sokolov reduction, J. Math. Phys. 39 (1998), 4729-4745, solv-int/9802013.

[10] Delduc F., Ragoucy E., Sorba P., Super-Toda theories and $W$-algebras from superspace Wess-ZuminoWitten models, Comm. Math. Phys. 146 (1992), 403-426.

[11] Evans J., Hollowood T., Supersymmetric Toda field theories, Nuclear Phys. B 352 (1991), 723-768.

[12] Evans J.M., Madsen J.O., Integrability versus supersymmetry, Phys. Lett. B 389 (1996), 665-672, hep-th/9608190.

[13] Ferreira L.A., Gervais J.-L., Guillén J.S., Saveliev M.V., Affine Toda systems coupled to matter fields, Nuclear Phys. B 470 (1996), 236-288, hep-th/9512105.

[14] Gervais J.-L., Saveliev M.V., Higher grading generalizations of the Toda systems, Nuclear Phys. B 453 (1995), 449-476, hep-th/9505047. 
[15] Gomes J.F., Schmidtt D.M., Zimerman A.H., Super-WZNW with reductions to supersymmetric and fermionic integrable models, Nuclear Phys. B $\mathbf{8 2 1}$ (2009), 553-576, arXiv:0901.4040.

[16] Gomes J.F., Starvaggi Franca G., de Melo G.R., Zimerman A.H., Negative even grade mKdV hierarchy and its soliton solutions, J. Phys. A: Math. Theor. 42 (2009), 445204, 11 pages, arXiv:0906.5579.

[17] Grigoriev M., Tseytlin A., Pohlmeyer reduction of $\mathrm{AdS}_{5} \times S^{5}$ superstring sigma model, Nuclear Phys. B 800 (2008), 450-501, arXiv:0711.0155.

[18] Inami T., Kanno H., Lie superalgebraic approach to super Toda lattice and generalized super KdV equations, Comm. Math. Phys. 136 (1991), 519-542.

[19] Madsen J.O., Miramontes J.L., Non-local conservation laws and flow equations for supersymmetric integrable hierarchies, Comm. Math. Phys. 217 (2001), 249-284, hep-th/9905103.

[20] Manin Yu.I., Radul A.O., A supersymmetric extension of the Kadomtsev-Petviashvili hierarchy, Comm. Math. Phys. 98 (1985), 65-77.

[21] Miramontes J.L., Tau-functions generating the conservation laws for generalized integrable hierarchies of KdV and affine Toda type, Nuclear Phys. B 547 (1999), 623-663, hep-th/9809052.

[22] Olshanetsky M.A., Supersymmetric two-dimensional Toda lattice, Comm. Math. Phys. 88 (1983), 63-76.

[23] Schmidtt D.M., Fermionic symmetry flows in non-Abelian Toda models, in preparation.

[24] Sorokin D.P., Toppan F., An $n=(1,1)$ super-Toda model based on OSp(1|4), Lett. Math. Phys. 42 (1997), 139-152, hep-th/9610038. 